\title{
Testing the magnetic flux paradigm for AGN radio loudness with a radio-intermediate quasar
}

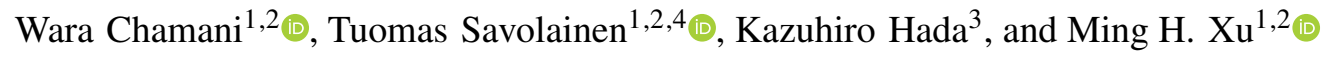 \\ 1 Aalto University Metsähovi Radio Observatory, Metsähovintie 114, 02540 Kylmälä, Finland \\ e-mail: wara.chamani@aalto.fi \\ 2 Aalto University Department of Electronics and Nanoengineering, PO Box 15500, 00076 Aalto, Finland \\ 3 Mizusawa VLBI Observatory, National Astronomical Observatory of Japan, 2-21-1 Owasa, Mitaka, Tokyo 181-8588, Japan \\ ${ }^{4}$ Max-Planck-Institut für Radioastronomie, Auf dem Hügel 69, 53121 Bonn, Germany
}

Received 26 February 2021 / Accepted 1 June 2021

\begin{abstract}
For understanding the diversity of jetted active galactic nuclei (AGN) and especially the puzzling wide range in their radio loudness, it is important to understand what role the magnetic fields play in setting the power of relativistic jets in AGN. We have performed VLBA phase-referencing observations of the radio-intermediate quasar III Zw 2 to estimate jet magnetic flux by measuring the coreshift effect. Multi-frequency observations at $4 \mathrm{GHz}, 8 \mathrm{GHz}, 15 \mathrm{GHz}$, and $24 \mathrm{GHz}$ were made using three nearby calibrators as reference sources. By combining the self-referencing core shift of each calibrator with the phase-referencing core shifts, we obtained an upper limit of 0.16 mas for the core shift between 4 and $24 \mathrm{GHz}$ in III Zw 2. By assuming equipartition between magnetic and particle energy densities and adopting the flux-freezing approximation, we further estimated the upper limit for both the magnetic field strength and poloidal magnetic flux threading the black hole. We find that the upper limit to the measured magnetic flux is smaller by at least a factor of five compared to the value predicted by the magnetically arrested disk (MAD) model. An alternative way to derive the jet magnetic field strength from the turnover of the synchrotron spectrum leads to an even smaller upper limit. Hence, the central engine of III Zw 2 has not reached the MAD state, which could explain why it has failed to develop a powerful jet even though the source harbours a fast-spinning black hole. However, it generates an intermittent jet, which is possibly triggered by small-scale magnetic field fluctuations, as predicted by the magnetic flux paradigm. We propose here that combining black hole spin measurements with magnetic field measurements from the very-long-baseline-interferometry core-shift observations of AGN over a range of jet powers could provide a strong test for the dominant factor that sets the jet power relative to the available accretion power.
\end{abstract}

Key words. galaxies: active - galaxies: jets - galaxies: magnetic fields - galaxies: Seyfert - astrometry techniques: high angular resolution

\section{Introduction}

One of the major enigmas regarding active galactic nuclei (AGN) involves their jet production efficiency that is, the ratio of jet power, $P_{\mathrm{j}}$, to accretion power, $\dot{M} c^{2}$, where $\dot{M}$ is the mass accretion rate and $c$ is the speed of light. It is not well understood how supermassive black holes (SMBHs) generate relativistic jets with a wide range of jet powers in sources with similar accretion powers. This issue is tangible in the observed spread (originally even dichotomy) of the radio-loudness parameter, defined as the ratio of the radio luminosity of an AGN to its optical luminosity at some specific frequencies (Strittmatter et al. 1980; Kellermann et al. 1989). Radio-loud quasars (RLQs) and radio-quiet quasars (RQQs) quasars can have a difference of at least four orders of magnitude in radio loudness - which is considered a proxy for the jet power - but show similar black hole (BH) masses and accretion rates (Sikora et al. 2007). We should note here that 'radio-quiet' sources are by no means 'radio-silent' and that AGN can dominate the radio emission in RQQ host galaxies at $z \sim 1$ (White et al. 2017). The radio emission related to accretion in RQQs could originate in small-scale 'aborted' jets (Ghisellini et al. 2004), in quasar-driven winds (Zakamska \& Greene 2014), or in a hot corona above the accretion disk (Raginski \& Laor 2016).
Possible explanations for the wide radio-loudness distribution include differences solely in BH spins (so-called spin paradigm; see e.g., Moderski et al. 1998; Sikora et al. 2007; Tchekhovskoy et al. 2010) or differences in the magnetic flux threading the $\mathrm{BH}$ combined with the variation in $\mathrm{BH}$ spins (so-called magnetic flux paradigm; Sikora \& Begelman 2013). These explanations are motivated by the fact that the currently favoured jet launching model uses magnetic fields to extract rotational energy from the spinning BH (Blandford \& Znajek 1977). Attributing the radio-loudness distribution solely to a $\mathrm{BH}$ spin distribution and its evolution seems to be, however, in contradiction with the expected cosmological evolution of spin in massive BHs (Volonteri et al. 2013). Moreover, it appears that not only radio-loud AGN but also radio-quiet AGN harbour high spinning BHs (Reynolds 2013, 2021).

Although accretion onto the SMBH is required for building up the launching jet in the first place, the level of the disk magnetisation in different environments might also set the different jet production efficiency of RLQs and RQQs (see Blandford et al. 2019). By highly efficient jet production we mean jet powers as high as the accretion power or even higher (Rawlings \& Saunders 1991; McNamara et al. 2011; Ghisellini et al. 2014). Such powerful relativistic jets can be ejected by the Blandford-Znajek process around a spinning BH that is threaded 
by a poloidal magnetic field with a maximum strength that a given accretion rate allows. This occurs when enough large-scale poloidal magnetic flux is accumulated in the inner accretion disk so that its magnetic pressure equals the ram pressure of the accretion flow. Such a state is referred to as a magnetically arrested disk (MAD; Narayan et al. 2003; Tchekhovskoy et al. 2011; McKinney et al. 2012).

The MAD scenario has been successfully tested with a sample of radio-loud AGN consisting of mostly blazars and nearby radio galaxies by Zamaninasab et al. (2014). Their results show that, for the majority of the sources, the observationally inferred jet magnetic flux is comparable to the maximum predicted magnetic flux ( $\sim 50$ in their dimensionless units) in the MAD state.

If the magnetic flux paradigm is the explanation for the observed wide range in radio loudness, then radio-quiet AGN should have magnetic fluxes that are generally well below the MAD level. In order to test this, it is necessary to study the magnetic field strengths in sources with weak jets but high BH spins as well as in recent RQQs that have transformed into RLQs, although with possibly short-lived or young jets (Nyland et al. 2020). Inferring the magnetic fluxes from observations for a diversity of sources can confirm whether the low jet production efficiency can indeed be attributed to the failure in the accumulation of enough magnetic flux to reach the MAD state.

Most of the available methods to measure jet magnetic field strengths are based on resolving the jet emission regions that are partially opaque due to synchrotron self-absorption (Marscher 1983). One way is to use high angular resolution, multifrequency radio imaging observations to measure the frequencydependent positional shift of the synchrotron photosphere along the jet and to assume an equipartition between energy densities of the radiating particles and magnetic fields in the jet (Lobanov 1998). The method requires sub-milliarcsecond imaging and astrometry with very-long-baseline interferometry (VLBI). The above technique, commonly known as the 'core-shift' measurement, has been increasingly used to infer jet magnetic field strengths over the past decade (Kovalev et al. 2008; O'Sullivan \& Gabuzda 2009; Sokolovsky et al. 2011; Pushkarev et al. 2012, 2018; Kutkin et al. 2014; Voitsik et al. 2018; Plavin et al. 2019). Another, closely related method for measuring the jet magnetic field strength is to use multi-frequency VLBI to resolve the size of the emission region at its synchrotron turnover frequency this gives the magnetic flux density without an equipartition assumption but tends to be more difficult in practice than the core-shift measurement (e.g., Savolainen et al. 2008; Hodgson et al. 2017). Jet magnetic fields can also be inferred by modelling the broadband spectral energy distribution, especially in blazars, although such estimates are usually quite model-dependent (see Hovatta \& Lindfors 2019, and references therein).

While the core-shift method has proved itself to be a robust way to measure magnetic field strengths in radio-loud AGN, it is challenging to make these measurements for weak jets in radio-quiet sources due to their faintness. One solution to this problem is to examine radio-intermediate quasars (RIQs), which are thought to be relativistically boosted counterparts of RQQs (Falcke et al. 1996a,b; Blundell \& Beasley 1998). A good example of such an RIQ is IIIZw 2, also known as Mrk 1501 and PG 0007+106. Its observed radio loudness $\left(\mathcal{R}=L_{5 \mathrm{GHz}} / L_{B}\right.$, where $L_{5 \mathrm{GHz}}$ is the radio luminosity at $5 \mathrm{GHz}$ and $L_{B}$ is the optical $B$-band luminosity of the nucleus) ranges from 150 to 200 (Falcke et al. 1996a; Sikora et al. 2007) and, accounting for the bulk Lorentz factor, places the source in or near the radio-quiet group with intrinsic radio loudness ranging from 2 to 35 (Chamani et al. 2020). This source has a compact,
Table 1. Phase reference sources for III Zw 2.

\begin{tabular}{lcc}
\hline \hline Source & $d^{(1)}\left(^{\circ}\right)$ & Redshift \\
\hline J0006+1235 & 1.91 & $0.98^{(2)}$ \\
J0007+1027 & 0.82 & - \\
J0008+1144 & 0.98 & - \\
\hline
\end{tabular}

Notes. ${ }^{(1)}$ Separation from the target. ${ }^{(2)}$ Snellen et al. (2002).

core-dominated, low-power jet, and it harbours a rapidly spinning BH (Chamani et al. 2020) with a mass of $1.8 \times 10^{8} M_{\odot}$ (Grier et al. 2012). The parsec-scale jet was ejected towards the north-western direction as observed at $43 \mathrm{GHz}$ in the late $1990 \mathrm{~s}$ (Brunthaler et al. 2000, 2005). At kiloparsec scales, a twosided structure has been detected; one of the components is a weak lobe or a hot spot connected to the core with a structure resembling a jet on the south-western side (Unger et al. 1987; Brunthaler et al. 2005; Cooper et al. 2007), and an even fainter lobe is seen on the north-eastern side (Brunthaler et al. 2005). In the late 1990s an episode of strong outbursts led to changes in the turnover frequency of the synchrotron spectrum, as well as to the ejection of a new emission feature in VLBI images moving with an apparent superluminal speed of $1.25 \mathrm{c} \pm 0.09 \mathrm{c}$ at $43 \mathrm{GHz}$ (Brunthaler et al. 2000). Two more ejections have been observed since then with the Very Long Baseline Array (VLBA) at $15 \mathrm{GHz}$ with apparent speeds of $1.58 \mathrm{c} \pm 0.29 \mathrm{c}$ and $1.358 \mathrm{c} \pm 0.074 \mathrm{c}$ (Lister et al. 2019).

In this work, we aim to test the magnetic flux paradigm and determine whether this particular RIQ has reached the MAD state or not. III Zw 2 has a relatively low jet production efficiency yet has a rapidly spinning $\mathrm{BH}$. Low accumulated magnetic flux would therefore be a natural expectation if the jets are launched by the Blandford-Znajek mechanism. We demonstrate here that such a test is feasible and can be carried out for a sample of RIQ and RQQ sources in the future. We estimate the magnetic field strength of III Zw 2, employing the coreshift measurements from phase-referencing observations. VLBA phase-referencing observations, although challenging, have been used to successfully measure the core shift, for example in M 87 (Hada et al. 2011) and NGC 4261 (Haga et al. 2015).

The paper is organised as follows: in Sect. 2, we present our phase-referencing multi-frequency VLBA observations, the data calibration, and imaging. Section 3 is devoted to the analysis method for measuring the core shift in III Zw 2. We present in Sect. 4 the results of the core-shift measurements, which include the self-referencing and the phase-referencing core shifts, the upper limit estimation of the core shift, and the magnetic field parameters for III Zw 2. Finally, Sect. 5 presents the summary and discussion.

\section{Observations and data calibration}

\subsection{Observations}

We carried out phase-referencing observations with the VLBA in order to do quasi-simultaneous multi-band relative astrometry of the target III Zw 2 with the nearby reference sources: $\mathrm{J} 0006+1235$, J0007+1027 and J0008+1144 (hereafter J0006, J0007 and J0008; see Table 1). Since III Zw 2 itself is very compact and bright enough for self-calibration, we use it to calibrate the phases, which are then transferred to the reference sources. Despite this, from here on we call the reference sources 'calibrators' for the sake of brevity. Two of the calibrators (J0007 
and J0008) are within $1^{\circ}$ from III Zw 2, while the third (J0006) is within $2^{\circ}$. The bright quasar $3 \mathrm{C} 454.3$ was observed as the primary calibrator.

The observations were performed on November 8, 2017, at four frequencies: $C$ band (centre frequency $4.148 \mathrm{GHz}$ ), $X$ band ( $7.652 \mathrm{GHz}), K_{U}$ band $(15.352 \mathrm{GHz}$; hereafter ' $U$ band') and $K$ band $(23.88 \mathrm{GHz})$. The use of the wide $C$ band receiver allowed the simultaneous observations at 4 and $8 \mathrm{GHz}$. The observed bandwidth was $256 \mathrm{MHz}$ at each band with eight $32 \mathrm{MHz}$ wide intermediate frequency (IF) sub-bands. The data were digitised with two bits, and dual polarisation was recorded at $U$ and $K$ bands, whereas for $C / X$ band only right-hand circular polarisation was recorded. The total recording rate was $2 \mathrm{Gbps}$.

The total observing time was eight hours. The observations were performed in frequency blocks of roughly $10 \mathrm{~min}, 16 \mathrm{~min}$, and $23 \mathrm{~min}$ each at $C / X, U$, and $K$ bands, respectively. The blocks were distributed evenly over the eight hours and each block consisted of fast switching between the target and the calibrators with cycle times adjusted for the each band and source. The cycle times (T-C-T) were 165-205 s at $C / X$ band, 135-160 s at $U$-band and $90-105 \mathrm{~s}$ at $K$-band. Total on-source observing times for III Zw 2 were $56 \mathrm{~min}$ at $K$-band, $36 \mathrm{~min}$ at $U$ band and $28 \mathrm{~min}$ at $C / X$ band.

Additionally, four geodetic-VLBI style $K$ band observing blocks of $30 \mathrm{~min}$ each were included in the schedule. The purpose of these 'geo-blocks' is to estimate the slowly varying component of the residual zenith tropospheric delay at each telescope by measuring the group delay of a number of compact sources over a range of azimuths and elevations in a rapid succession (Reid et al. 2009). We used the automatic geo-block generation algorithm implemented in the VLBI scheduling software SCHED with a list of candidate sources selected to have ICRF positions accurate to better than 1 mas and more than $100 \mathrm{mJy}$ of correlated flux on long baselines. To improve the delay measurement accuracy, we also used a geodetic-style frequency setup for the geo-blocks - the IFs were spread out to cover the whole available $500 \mathrm{MHz}$ band with minimally redundant frequency separations.

\subsection{Calibration}

We calibrated the data in AIPS (Greisen 2003) following standard procedures for a phase-referencing experiment (see for instance Reid et al. 2009). This included a priori corrections to the Earth Orientation Parameters and parallactic angle, corrections to dispersive ionospheric residual delays based on global maps of ionospheric total electron content derived from the Global Navigation Satellite System (GNSS) data, and calibration of instrumental single-band delays and phase offsets between IFs. We also corrected the small amplitude biases due to drifts in sampler threshold levels using autocorrelations, calibrated the complex bandpass shapes, and performed a priori amplitude calibration using recorded system temperatures and gain curves.

For processing the geodetic block data we used the procedure presented in Mioduszewski \& Kogan (2009). It consists of fringe-fitting the geo-block data, using the MBDLY task to measure the multi-band delays, and finally obtaining the zenith tropospheric delays and clock errors of the antennas from a fit to multi-band delays with the task DELZN. These solutions were applied to each dataset before the fringe-fitting. The fringefitting was performed with the task FRING on the target itself, assuming a point-like source, and then the solutions were applied with CLCAL to the calibrators, and phase-referenced images were obtained for each of them. We compared phase-referenced

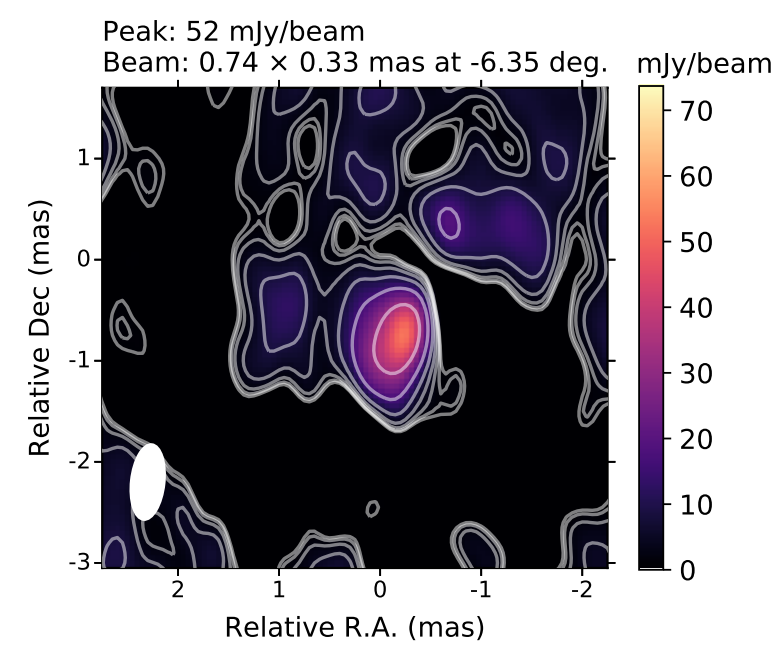

(a)

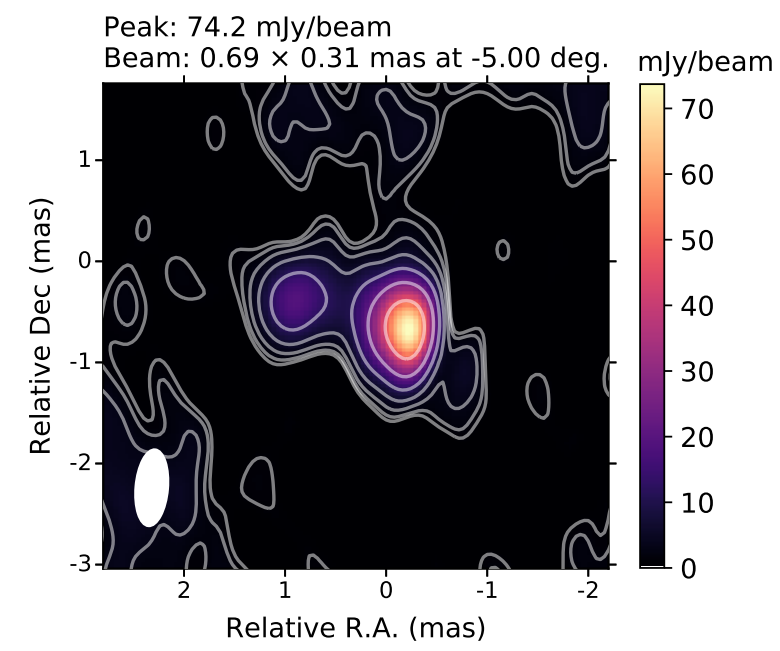

(b)

Fig. 1. Phase-referenced images of J0006 observed at the $K$-band after a few CLEAN iterations. (a) The image without troposphere delay corrections has a noise rms of $2.7 \mathrm{mJy} \mathrm{beam}^{-1}$ and (b) the image with troposphere delay corrections has noise rms of $1.3 \mathrm{mJy}_{\text {beam }}{ }^{-1}$. The interferometric beam (ellipse) is displayed on the bottom-left corner of each image. Contours represent $1 \%, 2 \%, 4 \%, 8 \%, 16 \%, 32 \%$, and $64 \%$ of the peak intensity at each image.

CLEAN images with and without tropospheric delay corrections, and we found that dynamic range of the images improved significantly with the tropospheric delay calibration, especially at the higher frequencies. On average, the signal-to-noise ratio ( $\mathrm{S} / \mathrm{N}$ or peak over noise rms) improvements were a factor of $1.5,1.4,2.1$, and 2.0 for the $C, X, U$ and $K$ bands, respectively. The most notable improvement of the $\mathrm{S} / \mathrm{N}$ by a factor of 3 was obtained for the $U$ and $K$ bands for J0006. An illustration of this is shown in Fig. 1, which shows the images of J0006 with and without troposphere correction at the $K$-band.

\subsection{Imaging}

We imaged the troposphere-corrected data with DIFMAP (Shepherd 1997) and produced both phase-referenced and selfcalibrated CLEAN images with natural weighting. These are all shown in Appendices A and B. III Zw 2 has a compact structure 


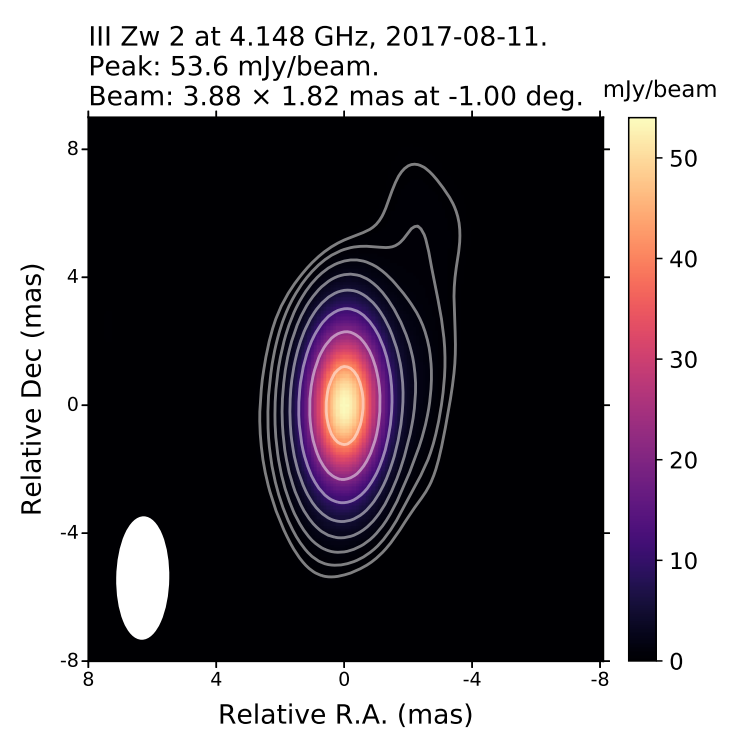

(a)

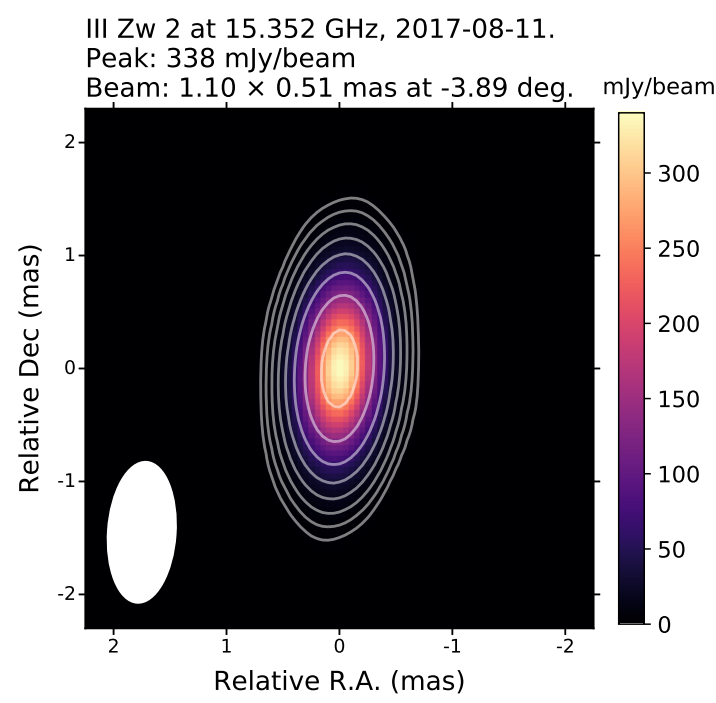

(c)

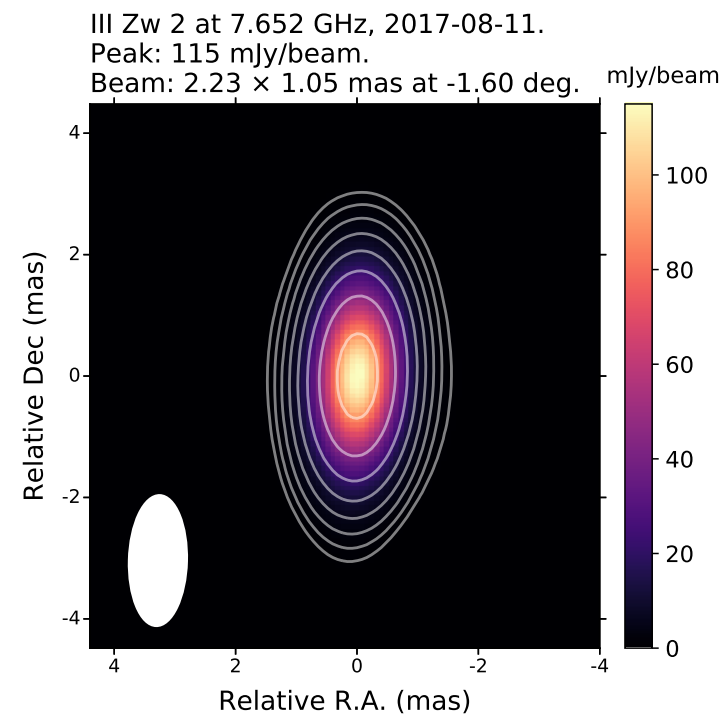

(b)

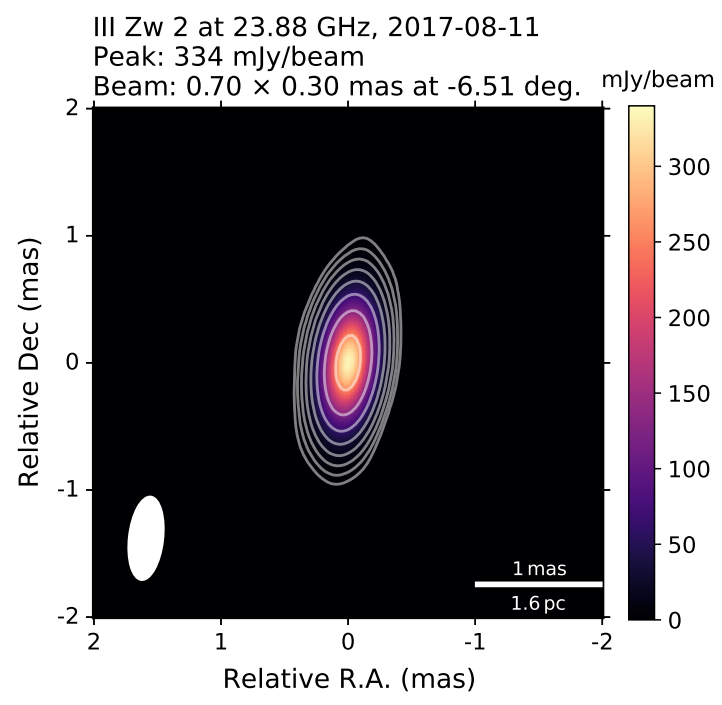

(d)

Fig. 2. Self-calibrated images of III Zw 2. The images were obtained at (a) $4.148 \mathrm{GHz}$, (b) $7.652 \mathrm{GHz}$, (c) $15.352 \mathrm{GHz}$ and (d) $23.88 \mathrm{GHz}$. The rms noise level from the lower to the higher frequency is $0.1,0.1,0.1$ and $0.2 \mathrm{mJy}_{\text {beam }}{ }^{-1}$. The interferometric beam (ellipse) is displayed on the bottom-left corner of each image. Contours represent $-0.6 \%, 0.6 \%, 1.2 \%, 2.4 \%, 4.8 \%, 9.6 \%, 19.2 \%, 38.4 \%$, and $76.8 \%$ of the peak intensity at each image.

with very little extended emission at all the bands (see Fig. 2). However, previous observations show that the jet is oriented towards the west-south-west at kiloparsec scales (Cooper et al. 2007) and to the west-north-west at parsec scales Pushkarev et al. (2017).

On the other hand, the CLEAN images of all the calibrators show extended jet emission at all frequencies. The most distant calibrator, J0006, has a jet oriented towards the east with a compact radio core seen at all frequencies and a bright component downstream, which is resolved at 15 and $24 \mathrm{GHz}$ (see Fig. B.1). The nearest calibrator $\mathrm{J} 0007$ has a rich and complex extended jet structure towards the north with a certain degree of bending visible at all frequencies. J0007 also has a compact core and a visible bright knot located farther north (see Fig. B.2). The second nearest calibrator J0008 has an extended jet structure oriented towards the south-east with a compact core (see Fig. B.3).
We point out here that our VLBA observations have produced, for the first time, high-dynamic-range, high-resolution images of the calibrators that show their parsec-scale radio morphology in detail.

The core spectrum of III Zw 2 and each calibrator was obtained by fitting a 2D Gaussian component directly to the visibility data. The description of the target spectrum is given in Sect. 4.4.2. Details of the core spectra of individual calibrators are provided in Appendix C.

\section{Analysis method}

The core is typically - although not always - the brightest and the most compact region found in the VLBI images. Its position $(r)$ from the central engine changes with the frequency $(v)$ due to opacity changes, becoming visible at the distance where 

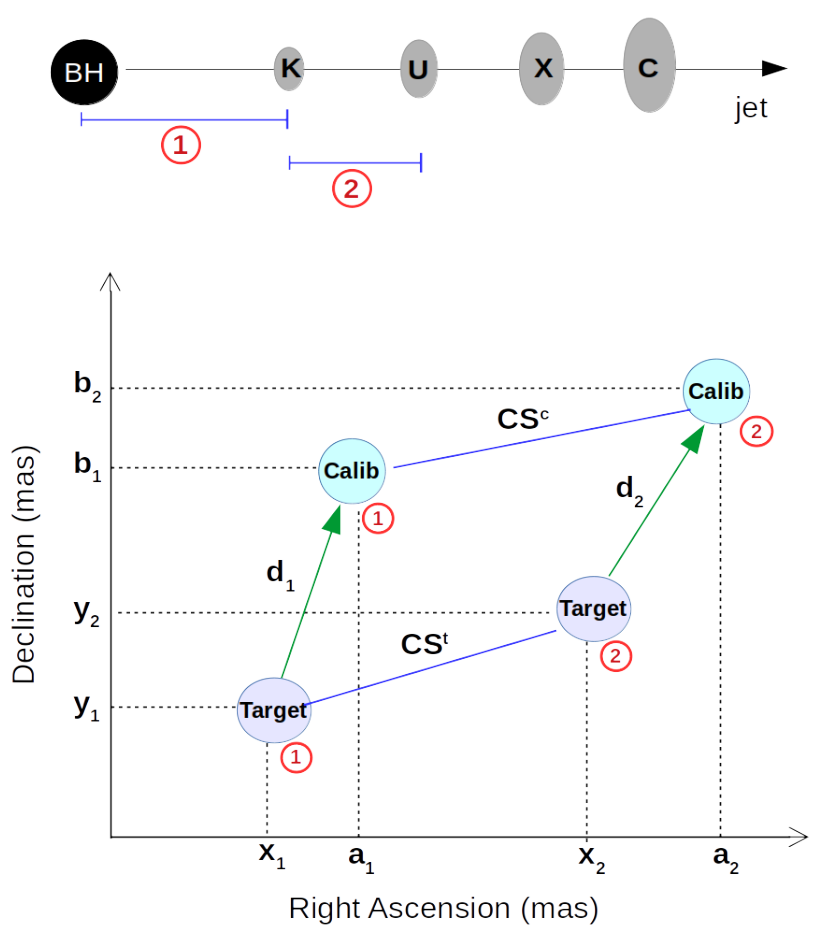

Fig. 3. Top: BH (filled black circle) that launches a jet in the horizontal direction. The different core positions at each frequency are shown in ellipses. The $K$-band core's distance from the BH is shown with the red circle ' 1 '. The distance between the adjacent frequencies $K$ and $U$ bands is shown with the red circle ' 2 '. Bottom: schematic diagram describing the phase-referencing positions $\left(d_{1}, d_{2}\right)$ and the core shifts of the target $\left(\mathrm{CS}^{t}\right)$ and a calibrator $\left(\mathrm{CS}^{c}\right)$. All coordinates are specified for each source.

$\tau \sim 1$ to synchrotron self-absorption. If one assumes that the magnetic field strength and the particle number density decrease with the distance along the jet as power laws $B=B_{1}\left(\frac{r_{1}}{r}\right)^{m}$ and $N=N_{1}\left(\frac{r_{1}}{r}\right)^{n}$, where $B_{1}$ and $N_{1}$ are the values at the distance of $r_{1}=1 \mathrm{pc}$, the dependence of the core position on the frequency follows $r \propto v^{-1 / k_{r}}$ (Blandford \& Königl 1979). Here $k_{r}$ depends on $m, n$ and the optically thin spectral index of the emission (see Eq. (1) in Lobanov 1998). The model of Blandford \& Königl assumes a supersonic, narrow, conical jet in equipartition with a constant half-opening angle and Lorentz factor, which would imply $k_{r}=1$. For such a jet, $m=1$ and $n=2$ are typically assumed. The measurement of the positional shift of the core as a function of frequency, together with the equipartition assumption, allows the measurement of the magnetic field strength and the particle number density.

In our study, we measure the core shifts of both the target and the calibrators since all calibrators are AGN with jets. Thus the measurement of the phase-referencing core shift of the target is actually a combination of the calibrators' own core shifts and the target's core shift. The latter is what we want to investigate. Figure 3 displays a diagram where the core positions and the core-shift vectors of the target and a calibrator are visualised. At position (1) when both the target and the calibrator are observed at the highest frequency band ' $K$ ', their coordinates are $x_{1}, y_{1}$ and $a_{1}, b_{1}$ respectively. The distance between the target and calibrator core in the phase-referencing map is denoted by $d_{1}$. At position (2) both the target and the calibrator are observed at the frequency band ' $U$ '. At this point their coordinates are $x_{2}, y_{2}$ and $a_{2}, b_{2}$ respectively. At this frequency the core position of the calibrator is $d_{2}$. The core shifts between the $K$ and $U$ bands are denoted as $\mathrm{CS}^{t}=\left(\begin{array}{l}C_{x}^{t} \\ C_{y}^{t}\end{array}\right)$ for the target and $\mathrm{CS}^{c}=\left(\begin{array}{l}C_{x}^{c} \\ C_{y}^{c}\end{array}\right)$ for the calibrator. For the calibrator, the positions are related by

$\left(\begin{array}{l}a_{1} \\ b_{1}\end{array}\right)+\left(\begin{array}{l}C_{x}^{c} \\ C_{y}^{c}\end{array}\right)=\left(\begin{array}{l}a_{2} \\ b_{2}\end{array}\right)$

and for the target,

$\left(\begin{array}{l}x_{1} \\ y_{1}\end{array}\right)+\left(\begin{array}{l}C_{x}^{t} \\ C_{y}^{t}\end{array}\right)=\left(\begin{array}{l}x_{2} \\ y_{2}\end{array}\right)$.

The distances $d_{1}$ (at the high frequency) and $d_{2}$ (at the low frequency) are given by

$d_{1}=\left(\begin{array}{l}d_{1 x} \\ d_{1 y}\end{array}\right)=\left(\begin{array}{l}a_{1} \\ b_{1}\end{array}\right)-\left(\begin{array}{l}x_{1} \\ y_{1}\end{array}\right)$,

and

$d_{2}=\left(\begin{array}{l}d_{2 x} \\ d_{2 y}\end{array}\right)=\left(\begin{array}{l}a_{2} \\ b_{2}\end{array}\right)-\left(\begin{array}{l}x_{2} \\ y_{2}\end{array}\right)$.

Subtracting Eqs. (1) and (2), plugging in (3) and (4), and rearranging, we obtain the core shift for the target as

$\left(\begin{array}{l}C_{x}^{t} \\ C_{y}^{t}\end{array}\right)=\left(\begin{array}{l}C_{x}^{c} \\ C_{y}^{c}\end{array}\right)+\left(\begin{array}{l}d_{1 x} \\ d_{1 y}\end{array}\right)-\left(\begin{array}{l}d_{2 x} \\ d_{2 y}\end{array}\right)$

Since all of our calibrators have extended jet structure, the core-shift vectors of the calibrator, $\mathrm{CS}^{c}$, for the given frequency pair $U K$ can be measured using the self-referencing method, namely, aligning optically thin jet components at different frequencies. The distances $d_{1}$ and $d_{2}$ can be measured from phasereferencing observations. We note that the method described above does not make any a priori assumption of the jet directions of the sources involved. The measurement of $\mathrm{CS}^{c}, d_{1}$ and $d_{2}$ are described in the next section.

\section{Results}

In this section, we describe separately the measurements of the self-referencing and phase-referencing core shifts.

\subsection{Self-referencing core shifts}

A well-known method for measuring the core shifts in VLBI images is so-called self-referencing. The method consists of aligning optically thin regions of the jet at the different frequencies (Lobanov 1998; Kovalev et al. 2008; Sokolovsky et al. 2011; Fromm et al. 2013). To achieve this, we used the 2D crosscorrelation technique (Walker et al. 2000; Croke \& Gabuzda 2008; Pushkarev et al. 2012; Fromm et al. 2013; Plavin et al. 2019 ) to align images at adjacent frequency pairs: $C X, X U$, and $U K$ for each calibrator. The cross-correlation analysis was performed with the software described in Pushkarev et al. (2012). For such an analysis, we first produced images at different frequencies with the same pixel size - the minor axis of the beam divided by 20 - and both convolved with the restoring beam of the lower frequency image. The image alignment procedure was run ten times per image pair by selecting slightly different optically thin features every time and excluding the core. The resulting spectral index maps and further details are given in Figs. E.1-E.3 in Appendix E. We point out that mean image shift values have been used for the core-shift estimations. 
Table 2. Self-referencing core-shift vectors of each calibrator for different frequency pairs.

\begin{tabular}{cccccc}
\hline \hline Source & $\begin{array}{c}\text { Frequency } \\
\text { pair }\end{array}$ & $\begin{array}{c}\text { RA } \\
(\mathrm{mas})\end{array}$ & $\begin{array}{c}\text { Dec } \\
\text { (mas) }\end{array}$ & $\begin{array}{c}\text { Absolute values } \\
\text { (mas) }\end{array}$ & $\begin{array}{c}\text { Projected absolute } \\
\text { values (mas) }\end{array}$ \\
\hline \multirow{3}{*}{$\mathrm{J} 0006$} & $C X$ & $0.49 \pm 0.06$ & $0.05 \pm 0.05$ & $0.49 \pm 0.06$ & $0.49 \pm 0.06$ \\
& $X U$ & $0.17 \pm 0.03$ & $0.07 \pm 0.03$ & $0.18 \pm 0.03$ & $0.18 \pm 0.03$ \\
& $U K$ & $0.07 \pm 0.02$ & $0.01 \pm 0.03$ & $0.07 \pm 0.02$ & $0.07 \pm 0.02$ \\
\hline \multirow{3}{*}{$\mathrm{J} 0007$} & $C X$ & $0.01 \pm 0.04$ & $0.44 \pm 0.06$ & $0.44 \pm 0.06$ & $0.44 \pm 0.06$ \\
& $X U$ & $-0.01 \pm 0.02$ & $0.15 \pm 0.03$ & $0.15 \pm 0.03$ & $0.15 \pm 0.03$ \\
& $U K$ & $-0.04 \pm 0.01$ & $0.06 \pm 0.02$ & $0.07 \pm 0.02$ & $0.06 \pm 0.02$ \\
\hline \multirow{3}{*}{$\mathrm{J} 0008$} & $C X$ & $0.32 \pm 0.10$ & $-0.12 \pm 0.06$ & $0.34 \pm 0.10$ & $0.33 \pm 0.10$ \\
& $X U$ & $0.10 \pm 0.03$ & $0.05 \pm 0.03$ & $0.11 \pm 0.03$ & $0.09 \pm 0.03$ \\
& $U K$ & $-0.01 \pm 0.02$ & $0.02 \pm 0.02$ & $0.02 \pm 0.02$ & $-0.01 \pm 0.02$ \\
\hline
\end{tabular}

Notes. ${ }^{(1)}$ The magnitude of the vector. ${ }^{(2)}$ The scalar product of the core-shift vector with the mean direction vector.

The core was modelled at each frequency (in DIFMAP) with a single circular Gaussian component. The estimation of the coreshift vector for a given frequency pair was performed similarly to the method described by Pushkarev et al. (2012). In their method, the core-shift coordinates result from the differences between the image shift and core position differences from the map centre of a pair of frequencies $\left(v_{1}, v_{2}\right)$. We used two different approaches to estimate the core shift's absolute value: (a) simply calculating the magnitude of the core-shift vector, (b) projecting the core-shift vectors onto the mean direction vector (meaning the average of the core-shift vectors) to reduce the effect of random errors on core-shift vector directions. In the latter, the absolute values are obtained from the core-shift vector's scalar product with the mean vector.

All measured self-referencing core-shift vectors and their absolute values for all the calibrators are listed in Table 2 . The absolute core-shift values measured by both approaches give very similar results. Figure $4 \mathrm{a}, \mathrm{c}$, and e displays the selfreferencing core-shift vectors and mean vectors for each calibrator. All absolute core shifts are significant, except for the $U K$ core shift in J0008.

The absolute values of the core shifts and the power-law fits are illustrated in Fig. 4b, d, and f. The fit results show $k_{r}$ indices below one in J0006 and $\mathrm{J} 0007$ and well below one in $\mathrm{J} 0008$. These results suggest that the jets in these sources may not be conical. Alternatively, the deviation of $k_{r}$ from unity may also indicate a deviation from the equipartition or that the compact jet in these sources is otherwise not strictly of Blandford \& Königl type. An example of the latter option is a model where the core at the two highest frequencies does not correspond to a synchrotron photosphere, but instead to a recollimation shock (see Dodson et al. 2017, and references therein). The position of such a shock is achromatic, and that could explain the absence of a core shift between 15 and $24 \mathrm{GHz}$ in $\mathrm{J} 0008$.

Furthermore, the total positional uncertainties of the selfreferencing core-shift measurement for each coordinate are calculated as

$\Delta \theta_{\text {total,core-shift }}=\sqrt{\Delta \theta_{2 \mathrm{DCC}}^{2}+\Delta \theta_{\text {core-position }}^{2}+\Delta \theta_{\text {core-ident }}^{2}}$,

where $\Delta \theta_{2 \mathrm{DCC}}$ is the error from $2 \mathrm{D}$ cross-correlation of optically thin jet regions at the two different frequencies. We aligned the images ten times - slightly varying the jet features to be compared - and obtained a statistical error associated with the mean image-shift from the scatter in the ten alignment attempts. Since the 2D cross-correlation software works in full pixel steps, the lower limit to the error is pixelsize $/ 2=\left(\theta_{\text {beam,min }} / 40\right)$ where $\theta_{\text {beam,min }}$ is the minor axis of beam. The errors are listed in Appendix D, Table D.1.

$\Delta \theta_{\text {core-position }}$ is the uncertainty in the core position derived from Gaussian model-fitting directly to the interferometric visibilities (that is fitting in the $(u, v)$ plane). Following Lampton et al. (1976) we estimate the positional errors by moving the core component about its best-fit position on a grid with a small step size, fixing its position and letting all the other parameters of the model to vary freely when minimising $\chi^{2}$. We then find an area on the grid around the best-fit position for which $\Delta \chi^{2}=\chi^{2}-\chi_{\min }^{2}<C_{p}^{\alpha}$, where $\chi_{\min }^{2}$ is the minimum $\chi^{2}$ corresponding to the best-fit position and $C_{p}^{\alpha}$ is the critical value of the $\chi^{2}$-distribution with $p$ degrees of freedom and $\alpha$ is the desired significance level. Following Lampton et al. (1976) $p$ is the number of free parameters in the Gaussian model after fixing the position of the core $(p=2$ for a single circular Gaussian). For the significance level we used $\alpha=0.32$, corresponding to $68 \%(1-\sigma)$ confidence level. The errors are listed in Table D.2.

$\Delta \theta_{\text {core-ident }}$ is the core identification error. This corresponds to the uncertainty in correctly locating the core in a source that has a jet that can blend with the core at our resolution. We estimate $\Delta \theta_{\text {core-ident }}$ as the difference between the core position from the $(u, v)$ plane Gaussian model-fitting and the brightness peak position in a super-resolved image. The core identification errors are given in Table D.3.

\subsection{Phase-referencing core shifts}

We used phase-referenced CLEAN images (no self-calibration applied) to measure the radio core positions of the individual calibrators. The measured core positions are represented by the distances ' $d_{1}$ ' and ' $d_{2}$ ' (see Sect. 3) between the calibrator and the target at two different frequencies. Additionally, a small correction in the target's core position was applied due to a slight offset of the target's peak flux from the map centre $(0,0)$ at each frequency. The model-fitting of the core was performed by identifying the brightest peak in the compact region. We fitted the peak with a single circular Gaussian component. We also tried fitting an elliptical Gaussian component, which, however, appears to be more affected by the emission from the optically thin jet. This is indicated by the systematically larger fitted flux densities for the elliptical Gaussians than for the circular ones.

Phase-referencing core-shift vectors, together with the absolute values, are listed in Table 3 . We found again that the different approaches pointed out in Sect. 4.1 produce almost 
W. Chamani et al.: Testing the magnetic flux paradigm for radio loudness of AGN

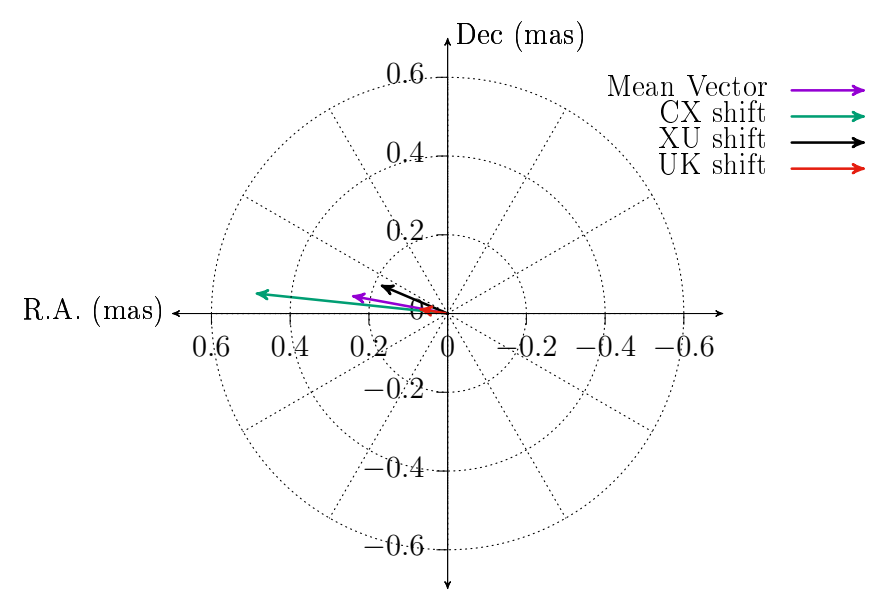

(a)

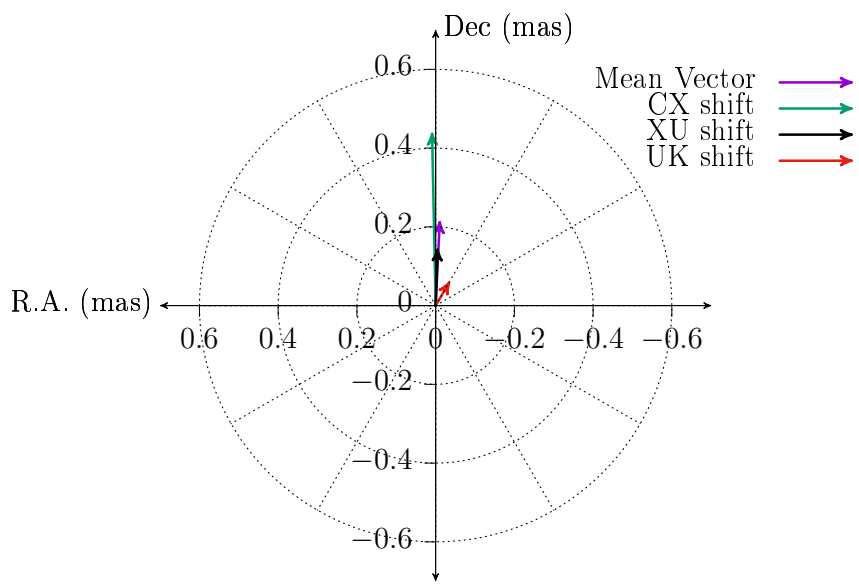

(c)

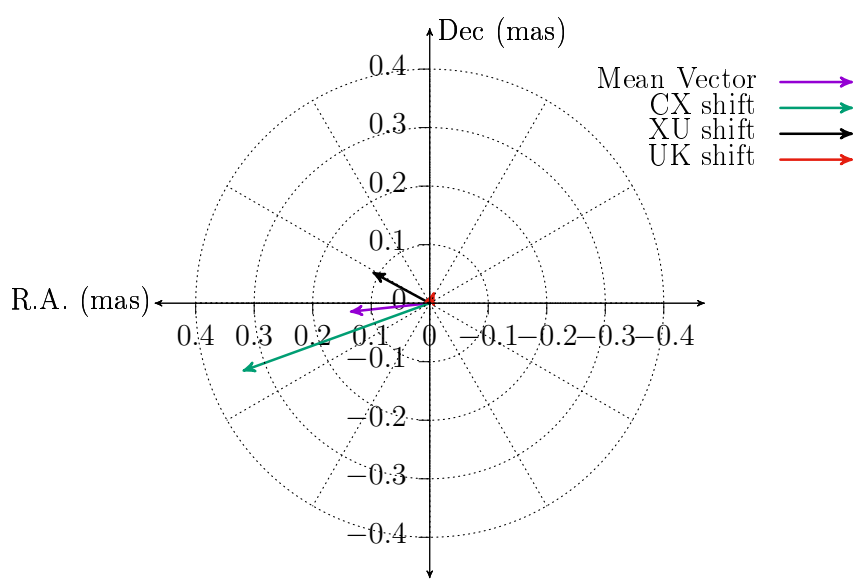

(e)

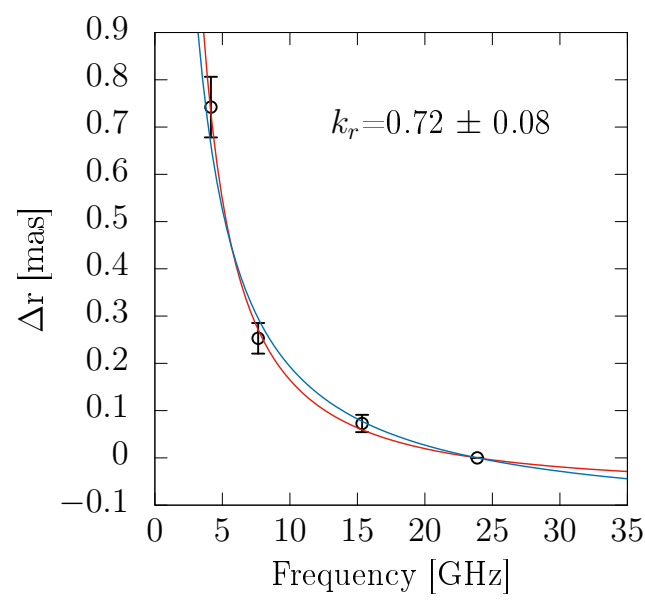

(b)

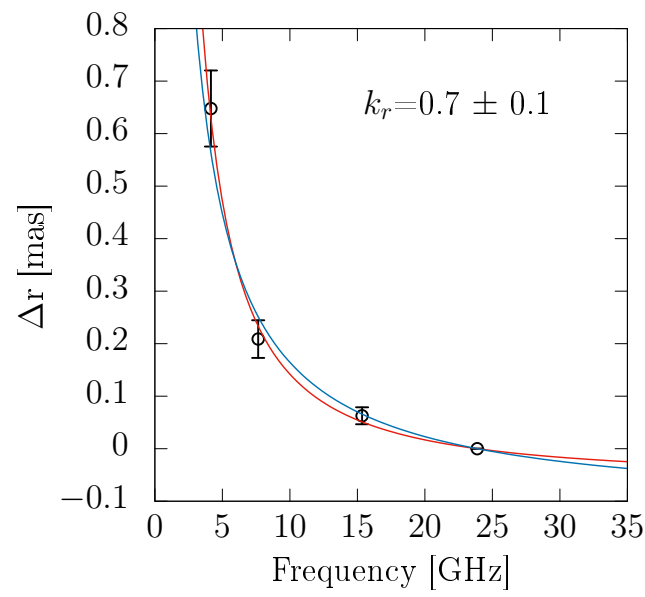

(d)

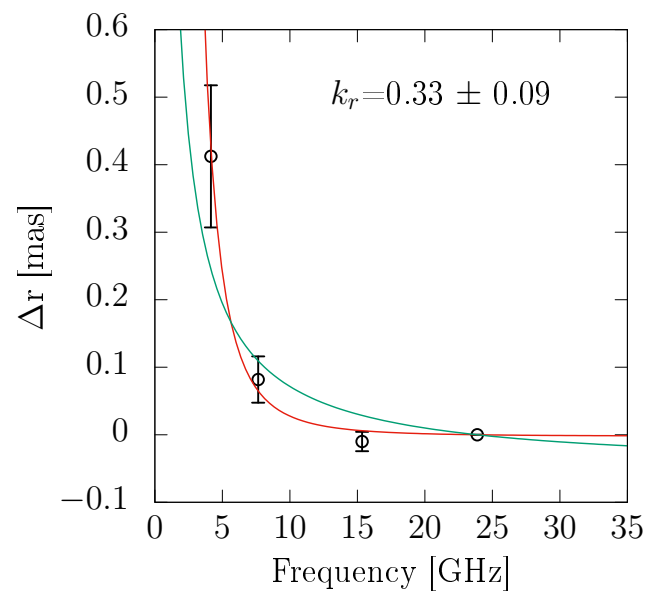

(f)

Fig. 4. Core-shift vectors and core-shift power-law fits for each calibrator. Left: self-referencing core-shift vectors in polar grids. The dotted lines are given at intervals of 30 degrees. (a) J0006: all vectors consistently point in the jet direction towards the east (see Fig. B.1). (c) J0007: $C X, X U$, and the mean vectors consistently point in the jet direction towards the north, while the $U K$ vector points towards the north-west (see Fig. B.2). (e) J0008: only the $C X$ and the mean vector consistently point in the jet direction towards the south-east (see Fig. B.3). Right: core shift ( $\Delta r)$ as a function of frequency $\left(v_{\mathrm{GHz}}\right)$. The red curve indicates the best fit of the form: $\Delta r=a\left(v_{\mathrm{GHz}}^{-1 / k_{r}}-23.88^{-1 / k_{r}}\right)$ with the $K$-band as the reference frequency. The blue and green curves indicate the fits when the power-law index $k_{r}$ is set to 1 with the fitting function: $\Delta r=b\left(v_{\mathrm{GHz}}^{-1}-23.88^{-1}\right)$. The constants $a$ and $b$ are fitting parameters. (b) J0006: the best fitting curve gives $a=5.8 \pm 1.4$ and $k_{r}=0.72 \pm 0.08$. The fit with $k_{r}=1$ gives $b=3.3 \pm 0.2$. (d) J0007: the best fitting curve gives $a=5.04 \pm 1.61$ and $k_{r}=0.71 \pm 0.11$. The fit with $k_{r}=1$ gives $b=2.8 \pm 0.2$. (f) J0008: the best fitting curve gives $a=31.7 \pm 38.8$ and $k_{r}=0.33 \pm 0.09$. Without the $U$-band, the fit with $k_{r}=1$ gives $b=1.2 \pm 0.4$. 
Table 3. Phase-referencing (combined) core-shift vectors for different frequency pairs.

\begin{tabular}{cccccc}
\hline \hline Source & $\begin{array}{c}\text { Frequency } \\
\text { pair }\end{array}$ & $\begin{array}{c}\text { RA } \\
(\mathrm{mas})\end{array}$ & $\begin{array}{c}\text { Dec } \\
\text { (mas) }\end{array}$ & $\begin{array}{c}\text { Absolute values } \\
\text { (mas) }\end{array}$ & $\begin{array}{c}\text { Projected absolute } \\
\text { values (mas) }\end{array}$ \\
\hline \multirow{3}{*}{$\mathrm{J} 0006$} & $C X$ & $0.38 \pm 0.04$ & $0.03 \pm 0.09$ & $0.38 \pm 0.04$ & $0.37 \pm 0.05$ \\
& $X U$ & $0.12 \pm 0.02$ & $0.08 \pm 0.04$ & $0.14 \pm 0.03$ & $0.13 \pm 0.02$ \\
& $U K$ & $0.10 \pm 0.02$ & $0.00 \pm 0.03$ & $0.10 \pm 0.02$ & $0.10 \pm 0.02$ \\
\hline \multirow{3}{*}{ 0007 } & $C X$ & $-0.02 \pm 0.03$ & $0.29 \pm 0.12$ & $0.29 \pm 0.12$ & $0.29 \pm 0.12$ \\
& $X U$ & $-0.04 \pm 0.03$ & $0.27 \pm 0.05$ & $0.27 \pm 0.04$ & $0.28 \pm 0.05$ \\
& $U K$ & $0.02 \pm 0.02$ & $0.09 \pm 0.02$ & $0.09 \pm 0.02$ & $0.09 \pm 0.02$ \\
\hline \multirow{2}{*}{ 0008 } & $C X$ & $0.27 \pm 0.07$ & $-0.27 \pm 0.08$ & $0.38 \pm 0.08$ & $0.37 \pm 0.09$ \\
& $X U$ & $0.16 \pm 0.02$ & $-0.07 \pm 0.05$ & $0.17 \pm 0.03$ & $0.17 \pm 0.04$ \\
& $U K$ & $0.03 \pm 0.02$ & $0.03 \pm 0.03$ & $0.04 \pm 0.03$ & $0.01 \pm 0.02$ \\
\hline
\end{tabular}

Notes. ${ }^{(1)}$ The magnitude of the vector. ${ }^{(2)}$ The scalar product of the core-shift vector with the mean direction vector.

identical absolute core-shift values. We note that to obtain the differences $d_{1}-d_{2}$ from Eq. (5), the notation of the core-shift vectors have to be changed to $X C, U X, K U$ with the coordinate sign changed.

The astrometric accuracy of phase referencing depends on random errors due to receiver noise and fast atmospheric phase fluctuations, as well as on various systematic effects that typically dominate. Since it is difficult to empirically estimate the systematic term, we build an error budget by theoretically evaluating each contributing factor. The sources of systematic errors include uncorrected residual delays due to (slowly varying) troposphere, ionosphere, source structure, instrumental effects, and errors in antenna and source positions. First-order corrections are made to most of these during the data calibration stage (see Sect. 2) and remaining systematics are further reduced by taking phase differences between the target and the calibrator and by taking position differences between frequency bands.

Switching between the target and a nearby calibrator effectively removes the residual instrumental delays (any that remain after clock corrections from geodetic blocks and manual phase calibration) and suppresses the atmospheric propagation delay errors proportionally to their separation on the sky (Reid \& Honma 2014; Thompson et al. 2017),

$\Delta \theta_{\mathrm{pos}} \simeq \theta_{\mathrm{sep}} \frac{|c \Delta \tau|}{B \sqrt{N}}$

where $\theta_{\text {sep }}$ is the angular distance between sources in radians (see Table 1), $c$ is the speed of light, $\Delta \tau$ is the residual atmospheric delay error, $B$ is the (longest) projected baseline length, and $N$ is the number of stations contributing to the long baselines of the array - in our case $N=5$ (Orosz et al. 2017). Furthermore, we analyse positional differences between frequency bands, which cancels all the non-dispersive delays that do not change on the timescale which the frequency bands are switched (the switching times are $0 \mathrm{~min}$ between $C$ and $X, \sim 13 \mathrm{~min}$ between $X$ and $U$, and $\sim 30 \mathrm{~min}$ between $U$ and $K$ ). This removes systematic uncertainties due to antenna position errors and source position errors, and it further suppresses errors due to the slowly varying component of the troposphere. Finally, since III Zw 2 is very compact at all the observed frequencies (see Fig. 2), we ignore the errors due to structural delays of the phase calibrator.

The remaining non-negligible terms in the positional error budget of the phase-referencing core shift are:

$\Delta \theta_{\text {total,pos }}=\sqrt{\Delta \theta_{\text {thermal }}^{2}+\Delta \theta_{\text {core-ident }}^{2}+\Delta \theta_{\text {trop }}^{2}+\Delta \theta_{\text {ion }}^{2}}$, where $\Delta \theta_{\text {thermal }}$ is the positional uncertainty due to random (thermal) errors evaluated as $\theta_{\text {beam }} /(2 \cdot S / N)$, where $\theta_{\text {beam }}$ is the beam size and $\mathrm{S} / \mathrm{N}$ is the peak flux of the phase-referenced image divided by the rms image noise. Since the beam is nearly northsouth-oriented, we use the minor axis FHWM of the beam, $\theta_{\text {beam,min }}$, as the beam size for RA and the major axis FWHM of the beam, $\theta_{\text {beam,max }}$, for Dec. The thermal errors are listed in Appendix D, Table D.4.

$\Delta \theta_{\text {core-ident }}$ is the core identification error. The evaluation of this error was done similarly as for the self-referencing (see the previous subsection). The core identification errors are given in Table D.5.

$\Delta \theta_{\text {trop }}$ is the positional uncertainty due to the tropospheric residual delays. These are discussed in detail below.

$\Delta \theta_{\text {ion }}$ is the positional uncertainty due to ionospheric residual delays. These are discussed in detail below.

Phase referencing suppresses the fast varying (wet) component of the troposphere and remaining errors due to this component are reflected in $\Delta \theta_{\text {thermal }}$. Slowly varying spatial tropospheric delay component is solved from the geodetic blocks and the remaining errors in phase referencing are due to uncertainties in the tropospheric delay gradient between the target and the calibrator after the geodetic block calibration. Since we are interested in positional differences between observing bands and since tropospheric delay is non-dispersive, only the timevariable part of the gradient error remains:

$\Delta \tau_{\text {trop }}^{v_{2}-v_{1}} \approx \Delta t \frac{\mathrm{d} \tau_{\text {zenith }}}{\mathrm{d} t} \sec (z) \tan (z)$

Here $\Delta t$ is the time interval between the change of the observing bands, $\frac{\mathrm{d} \tau_{\text {zenith }}}{\mathrm{d} t}$ is the time derivative of the residual zenith delay (after correction by geodetic blocks) and $z$ is the zenith angle. We (very) conservatively estimate that $\frac{\mathrm{d} \tau_{\text {zenith }}}{\mathrm{d} t}$ is of the same order as the time-derivative of the residual zenith delay solved from the geodetic blocks. We have calculated the average $\Delta \tau_{\text {trop }}^{v_{2}-v_{1}}$ for the telescopes participating in the longest baselines in E-W (MK-SC) and N-S (BR-SC) for elevations above 20 degrees. The resulting errors from combining $\Delta \tau_{\text {trop }}^{\nu_{2}-v_{1}}$ for the both telescopes of a baseline and using Eq. (7) are given in Table D.6.

As mentioned in Sect. 2, we corrected the dispersive ionospheric delays using global maps of ionospheric total electronic content (TEC) determined from GNSS observations. The analysis centres of the International GNSS Service produce these maps and make them publicly available. The global maps from the Jet Propulsion Laboratory (JPL) analysis centre were 
Table 4. Core-shift vectors of III Zw 2 with each calibrator.

\begin{tabular}{|c|c|c|c|c|c|c|c|}
\hline \multirow[t]{2}{*}{ Reference source } & \multicolumn{2}{|c|}{$C X$} & \multicolumn{2}{|c|}{$X U$} & \multicolumn{2}{|c|}{$U K$} & \multirow{2}{*}{$\begin{array}{c}C K \text { (upper limit) } \\
\text { Absolute value } \\
\text { (mas) }\end{array}$} \\
\hline & $\begin{array}{c}\text { RA } \\
\text { (mas) }\end{array}$ & $\begin{array}{c}\text { Dec } \\
\text { (mas) }\end{array}$ & $\begin{array}{c}\text { RA } \\
\text { (mas) }\end{array}$ & $\begin{array}{c}\text { Dec } \\
\text { (mas) }\end{array}$ & $\begin{array}{c}\text { RA } \\
\text { (mas) }\end{array}$ & $\begin{array}{c}\text { Dec } \\
\text { (mas) }\end{array}$ & \\
\hline J0006 & $0.11 \pm 0.07$ & $0.02 \pm 0.10$ & $0.05 \pm 0.03$ & $0.00 \pm 0.05$ & $-0.03 \pm 0.03$ & $0.01 \pm 0.04$ & $0.14 \pm 0.08(<0.22)$ \\
\hline J0007 & $0.03 \pm 0.05$ & $0.15 \pm 0.14$ & $0.03 \pm 0.04$ & $-0.13 \pm 0.06$ & $-0.05 \pm 0.02$ & $-0.03 \pm 0.03$ & $0.00 \pm 0.13(<0.13)$ \\
\hline J0008 & $0.05 \pm 0.12$ & $0.15 \pm 0.10$ & $-0.06 \pm 0.04$ & $0.12 \pm 0.06$ & $-0.04 \pm 0.02$ & $-0.01 \pm 0.04$ & $0.27 \pm 0.12(<0.39)$ \\
\hline Weighted mean & $0.06 \pm 0.04$ & $0.10 \pm 0.07$ & $0.01 \pm 0.02$ & $0.00 \pm 0.03$ & $-0.04 \pm 0.02$ & $-0.01 \pm 0.02$ & $0.09 \pm 0.07(<0.16)$ \\
\hline Absolute value of the weighted mean & \multicolumn{2}{|c|}{$0.12 \pm 0.06$} & \multicolumn{2}{|c|}{$0.01 \pm 0.02$} & \multicolumn{2}{|c|}{$0.04+0.02$} & $0.17 \pm 0.07(<0.24)$ \\
\hline Projected value of the weighted mean & \multicolumn{2}{|c|}{$0.11 \pm 0.07$} & \multicolumn{2}{|c|}{$0.00 \pm 0.03$} & \multicolumn{2}{|c|}{$-0.03 \pm 0.03$} & $0.09 \pm 0.08(<0.17)^{(\dagger)}$ \\
\hline
\end{tabular}

Notes. The absolute values for the $C K$ core shifts are calculated as $r=\sqrt{(C X+X U+U K)_{\mathrm{RA}}^{2}+(C X+X U+U K)_{\text {Dec }}^{2}}$. The Table contains rounded values. ${ }^{(\dagger)}$ Value from Fig. 5 b.

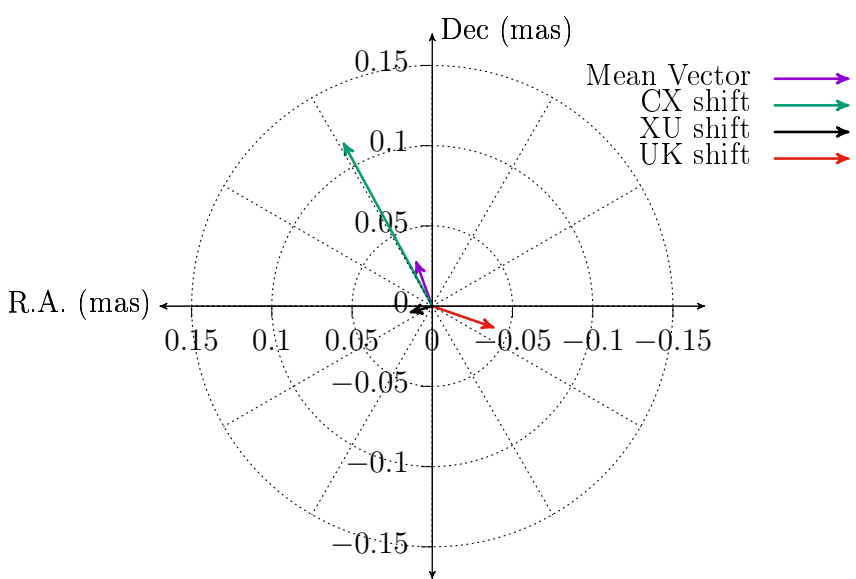

(a)

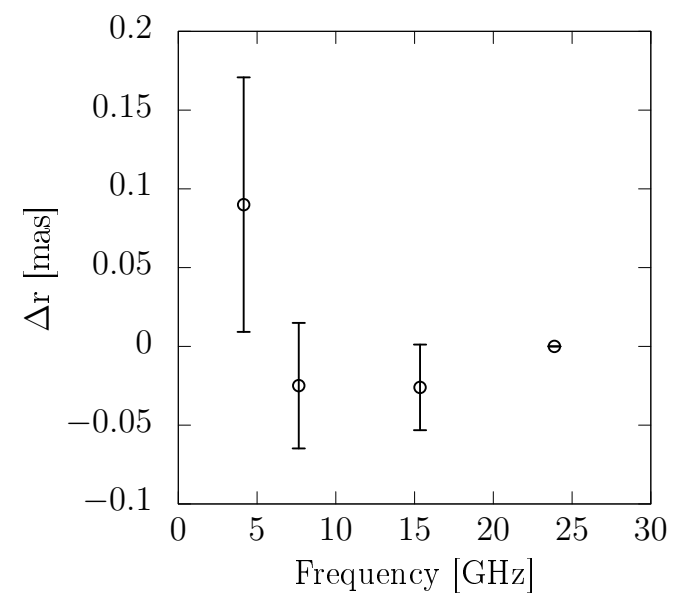

(b)

Fig. 5. Core-shift vectors and core-shift power-law fit for III Zw2. (a) Weighted mean core-shift vectors and mean direction vector of III Zw 2 combining all calibrators. (b) Projected core-shift values with $K$ band as the reference frequency using the weighted mean core-shift vectors of Table 4. The values relative to $K$ band are $0.089 \pm 0.081$ mas for $C K,-0.025 \pm 0.040$ mas for $X K$, and $-0.026 \pm 0.027$ mas for $U K$.

used in our data processing to calibrate the ionospheric effects. The maps from JPL have a latitude/longitude resolution of 2.5/5.0 degrees and a temporal resolution of $2.0 \mathrm{~h}$, and they are treated as a thin spherical layer at the height of $450 \mathrm{~km}$. Both slant TEC values and their uncertainties can be obtained from the global TEC maps. In order to derive the slant TEC values for the actual VLBA observations from these maps, we calculated the pierce point at the single layer of the TEC maps for each individual observation. We then determined the vertical TEC at the pierce point at the observing time by interpolating from the TEC maps both in space and in time, and mapped the vertical TEC to the line of sight by using the modified single layer mapping function. The uncertainties for slant TEC values were obtained based on the error propagation. $\Delta \theta_{\text {ion }}$ was derived for RA and Dec by considering slant TEC errors on the longest E-W and $\mathrm{N}$ $\mathrm{S}$ baselines, MK-SC and BR-SC, respectively. We calculated the average slant TEC errors for scans with source elevation above 20 degrees and used the relation

$\left|c \Delta \tau_{\text {ion }}\right|=40.3 \Delta I_{e} v^{-2}$,

where $\Delta I_{e}$ is the slant TEC uncertainty in TEC units (1 TECU corresponds to an electron column density of $10^{16} \mathrm{~m}^{-2}$ ) and $v$ is the observing frequency (Reid \& Honma 2014). The results combining Eqs. (7) and (10) are in Table D.7.

\subsection{Core shift of IIIZw2}

To obtain the core-shift vectors of the target III Zw 2, we use Eq. (5), which requires both the self-referencing and phasereferencing core-shifts vectors. Combining the results from Tables 2 and 3 we obtained the core-shift vectors of the target, listed in Table 4. Given that the errors are equal or even larger than the measured core-shift coordinates, we combined the measurements from all three calibrators and calculated their weighted mean core-shift vectors for each frequency pair. Furthermore, we also calculated the weighted mean core-shift vectors projection onto the mean direction vector, as illustrated in Fig. 5a.

The results show a disparity of the core-shift vector directions, and none of them points in the target's expected jet direction (towards the west). A similar result was found for the projected core shifts with individual calibrators (see Appendix F, Figs. F.1-F.3). These results imply that the core shift of III Zw 2 is very small compared to our measurement accuracy, which is evident in the vectors' random orientations (see Fig. 5b).

It is clear that there is a larger uncertainty of the core shift along the calibrators jet direction, which implies that the self-referencing core shifts and/or the calibrator core locations along the jet have systematic errors. Combining all three calibrators partly helps to mitigate these issues since they have different jet directions. However, we note that there is an overall eastward 
Table 5. Physical properties of III Zw 2.

\begin{tabular}{lccccccccc}
\hline \hline$z$ & $\begin{array}{c}D_{L} \\
(\mathrm{Mpc})\end{array}$ & $\beta_{\mathrm{app}}$ & $\delta$ & $\Gamma$ & $\begin{array}{c}\theta \\
(\mathrm{deg})\end{array}$ & $\begin{array}{c}\theta_{\mathrm{j}} \\
(\mathrm{deg})\end{array}$ & $\begin{array}{c}M_{\mathrm{BH}} \\
\left(M_{\odot}\right)\end{array}$ & $a$ & $\begin{array}{c}L_{\mathrm{acc}} \\
\left(\mathrm{erg} \mathrm{s}^{-1}\right) \\
(10)\end{array}$ \\
\hline 0.0898 & 405.5 & 1.58 & 1.7 & 1.9 & $35<\theta<55$ & $6.7<\theta_{\mathrm{j}}<9.5$ & $1.84 \pm 0.27 \times 10^{8}$ & $\geq 0.98$ & $8.1 \times 10^{44}, 2.5 \times 10^{45}$ \\
\hline
\end{tabular}

Notes. Column definitions: (1) redshift, Hernán Caballero (2012), (2) luminosity distance from Wright (2006), (3) apparent velocity from Lister et al. (2019), (4) Doppler factor from Hovatta et al. (2009), (5) Lorentz factor; $\Gamma=\frac{\beta_{\text {app }}^{2}+\delta^{2}+1}{2 \delta}$, (6) viewing angle range from Brunthaler et al. (2000), Hovatta et al. (2009) and Chamani et al. (2020), (7) intrinsic half-opening angle range inferred from Pushkarev et al. (2017), (8) BH mass from Grier et al. (2012), (9) BH spin from Chamani et al. (2020), (10) accretion disk luminosity values from Berton et al. (2015) and Falcke et al. (1995), respectively.

bias in the jet directions of the calibrators, which may affect the combined core shift.

As is evident from Table 4 and Fig. 5, we can only estimate an upper limit to the core-shift in III Zw 2. We consider two approaches to calculate the upper limit: (a) using the vector component perpendicular to the calibrator's jet direction. For that, we use J0007 for the RA component and J0006 for the Declination component (see Table 4). This gives a 1- $\sigma$ upper limit of $<0.15$ mas $(0.03 \pm 0.12$ mas $)$. Approach (b) consists of using the weighted mean or the projected value of the weighted mean coreshift between 4 and $24 \mathrm{GHz}$ in Table 4 . These give 1- $\sigma$ upper limits of $<0.16$ mas and $<0.17$ mas, respectively. The projected weighted mean is not a very useful quantity when all the coreshift vectors point in different directions like in III Zw 2. Therefore, we adopt the upper limit of $<0.16$ mas for the jet magnetic field strength calculations.

\subsection{Estimation of magnetic field parameters}

\subsubsection{Magnetic field strength from the core shift}

Assuming that the magnetic and (radiating) particle energy densities are in equipartition in the jet of III Zw 2, we can calculate $B_{1}$, the magnetic field strength in the jet ${ }^{1}$ (Lobanov 1998). The equipartition assumption together with jet's conical shape also implies that the core-shift index $k_{r}=1$ and that the mean jet frame magnetic field is dominated by the azimuthal component on parsec scales. Furthermore, we adopt the flux freezing approximation, which states that the poloidal magnetic flux threading the parsec-scale jet is equal to the poloidal flux threading the BH. Following Zamaninasab et al. (2014) we can then estimate an upper limit to the poloidal magnetic flux threading the horizon of the central $\mathrm{BH}$ in III Zw 2. In the following, we described briefly the observed parameters and relationships needed to estimate the jet magnetic field strength at $1 \mathrm{pc}$ as well as the jet magnetic flux.

Table 5 presents the physical parameters of III Zw 2 collected from the literature (see the references below the table). The parameters include the redshift $z$ and luminosity distance $D_{L}$, the apparent velocity, $\beta_{\text {app }}$, the Doppler factor $\delta$, the bulk Lorentz factor $\Gamma$, the viewing angle $\theta$, the half-opening angle $\theta_{\mathrm{j}}$ of the jet, the $\mathrm{BH}$ mass $M_{\mathrm{BH}}$, the spin $a$ and the accretion luminosity $L_{\mathrm{acc}}$. To estimate the range of values for $\theta_{\mathrm{j}}$, we first took the apparent full opening angle of 23.2 degrees measured by Pushkarev et al. (2017). The intrinsic half-opening angle was calculated as $\tan \left(\theta_{\mathrm{j}}\right)=\tan \left(23.2^{\circ} / 2\right) \sin \theta$ (Pushkarev et al. 2017). This results

\footnotetext{
${ }_{1} B_{1}$ corresponds to $B$ at a distance of $1 \mathrm{pc}$ from the jet apex, but the power-law dependence of $B$ on the distance along the jet allows one to calculate $B$ at any distance as long as the jet can be described by the Blandford-Königl model.
}

in a range of values of $6.7^{\circ}<\theta_{\mathrm{j}}<9.5^{\circ}$ and $0.22<\Gamma \theta_{\mathrm{j}}<0.32$ since $35^{\circ}<\theta<55^{\circ}$. $\Gamma \theta_{\mathrm{j}}$ agrees well with the typical value derived by Clausen-Brown et al. (2013).

Following Lobanov (1998), Hirotani (2005), Zamaninasab et al. (2014) and the corrections pointed out by Zdziarski et al. (2015), the core's offset $\Omega_{r v}$ and the magnetic field strength in equipartition $B_{1 \mathrm{pc}}^{\mathrm{eq}}$ at $1 \mathrm{pc}$ from the apex of the jet are given by the following relations:

$\Omega_{r v}=4.85 \times 10^{-9} \frac{\Delta r_{\text {mas }} D_{L}}{(1+z)^{2}} \frac{v_{1}^{1 / k_{r}} v_{2}^{1 / k_{r}}}{v_{2}^{1 / k_{r}}-v_{1}^{1 / k_{r}}}\left[\mathrm{pc} \mathrm{GHz}^{1 / k_{r}}\right]$,

where $v_{1}$ and $v_{2}$ are the observed frequencies (in GHz) with $v_{2}>$ $v_{1}, D_{L}$ is in pc and $\Delta r_{\text {mas }}$ is the core shift in mas between two frequencies $v_{1}$ and $v_{2}$. We use the same cosmology as Pushkarev et al. (2012) with $H_{0}=71 \mathrm{~km} \mathrm{~s}^{-1} \mathrm{Mpc}^{-1}, \Omega_{\mathrm{m}}=0.27$ and $\Omega_{\Lambda}=$ 0.73 . The magnetic field strength is then given by

$B_{1 \mathrm{pc}}^{\mathrm{eq}} \approx 0.025\left[\frac{\sigma_{\text {rel }} \Omega_{r v}^{3 k_{r}}(1+z)^{3}}{\delta^{2} \theta_{\mathrm{j}} \sin ^{3 k_{r}-1} \theta}\right]^{\frac{1}{4}} \quad[\mathrm{G}]$,

where $\sigma_{\text {rel }}$ is the ratio of magnetic and particle energy densities, which we assume to be unity. The above formula also assumes implicitly that the electron energy distribution has a power-law index of $p=2$, which corresponds to an optically thin spectral index of $\alpha=-0.5$ and that $\gamma_{\max } / \gamma_{\min }=10^{4.34}$. Setting $k_{r}=1$ (conical jet and equipartition) and inserting the core-shift upper limit of 0.16 mas with the range of values given for $\theta$ and $\theta_{\mathrm{j}}$, we calculated upper limits to $\Omega_{r v}$ and $B_{1 \mathrm{pc}}^{\mathrm{eq}}$ (see Table 6). The resulting mean jet frame magnetic field strength is below $60 \mathrm{mG}$.

On the other hand, the magnetic field strength at $1 \mathrm{pc}$ can be also calculated without assuming equipartition if we have a measurement of the core flux density (Zdziarski et al. 2015):

$B_{1 \mathrm{pc}}^{\text {noeq }} \simeq \frac{3.35 \times 10^{-11} D_{L} \Delta r_{\mathrm{mas}}^{5} \delta \tan ^{2} \theta_{\mathrm{j}}}{\left(v_{1}^{-1}-v_{2}^{-1}\right)^{5}[(1+z) \sin \theta]^{3} F_{v}^{2}}[\mathrm{G}]$,

where $F_{v}$ (in Jy) is the flux density in the flat part of the spectrum, $F_{v} \propto v^{0}$. For III Zw 2 the flat part of the spectrum lies between 15 and $24 \mathrm{GHz}$ (see Fig. 6) with a flux density of $0.34 \mathrm{Jy}$. Using the upper limit of the core shift between 4 and $24 \mathrm{GHz}$, the upper limit of $B_{1 \mathrm{pc}}^{\text {noeq }}$ is $4 \mathrm{mG}$. This is more than an order of magnitude smaller than the upper limit to $B_{1 \mathrm{pc}}^{\mathrm{eq}}$. Dividing Eq. (13) by Eq. (12) we see that the ratio $B_{1 \mathrm{pc}}^{\text {noeq }} / B_{1 \mathrm{pc}}^{\mathrm{eq}} \propto \Delta r^{17 / 4}$ for $k_{r}=1$. Therefore, the discrepancy between $B_{1 \mathrm{pc}}^{\text {noeq }}$ and $B_{1 \mathrm{pc}}^{\mathrm{eq}}$ only increases if the true core-shift value is less than our upper limit. This rises a possibility of a departure from the equipartition conditions in the jet of III Zw 2. However, we would need to have an actual measurement - instead of an upper limit - of the core shift in III Zw 2 to confirm this. 
Table 6. Parameters for the magnetic field of III Zw 2, using the coreshift upper limit of 0.16 mas; and the synchrotron self-absorption magnetic field strength, $B_{\mathrm{SSA}}$.

\begin{tabular}{lcc}
\hline \hline Parameter & Value & Units \\
\hline$\Omega_{r v}$ & $\leq 1.3$ & $\mathrm{pc} \mathrm{GHz}$ \\
$B_{1 \mathrm{pc}}^{\text {eq }}$ & $\leq 60$ & $\mathrm{mG}$ \\
$B_{1 \mathrm{pc}}^{\text {noeq }}$ & $\leq 4$ & $\mathrm{mG}$ \\
$B_{1 \mathrm{pc}, \mathrm{SSA}}$ & $\leq 20$ & $\mathrm{mG}$ \\
$\Phi_{\text {jet }}^{\mathrm{eq}}$ & $\leq 10^{32}$ & $\mathrm{G} \mathrm{cm}^{2}$ \\
$\Phi_{\text {jeeq }}^{\text {noeq }}$ & $\leq 7 \times 10^{30}$ & $\mathrm{G} \mathrm{cm}^{2}$ \\
$\Phi_{\text {jet,SSA }}$ & $\leq 3.6 \times 10^{31}$ & $\mathrm{G} \mathrm{cm}^{2}$ \\
$\Phi_{\mathrm{BH}, \mathrm{MAD}}{ }^{(a)}$ & $\sim 5 \times 10^{32}$ & $\mathrm{G} \mathrm{cm}^{2}$ \\
$\Phi_{\mathrm{BH}, \mathrm{MAD}}{ }^{(b)}$ & $(7-9) \times 10^{32}$ & $\mathrm{G} \mathrm{cm}^{2}$ \\
$\Phi_{\mathrm{BH}, \mathrm{MAD}}{ }^{(c)}$ & $1.1 \times 10^{33}$ & $\mathrm{G} \mathrm{cm}^{2}$ \\
\hline
\end{tabular}

Notes. ${ }^{(a)}$ With the average value of $L_{\text {acc }}$ (values taken from Table 5) and adopting $\eta=0.4{ }^{\left({ }^{b}\right)}$ With the average value of $L_{\text {acc }}$ and adopting $\eta=0.13-0.19$ from Davis \& Laor (2011). ${ }^{(c)}$ Using the $L_{\text {acc }}$ value from Falcke et al. (1995) and adopting $\eta=0.13$ from Davis \& Laor (2011).

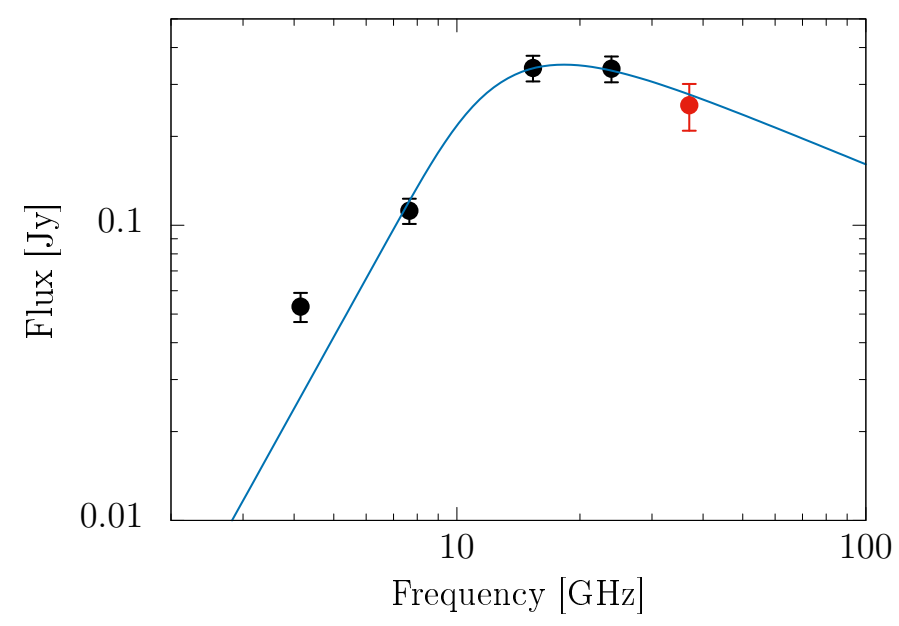

Fig. 6. Correlated flux density of III Zw 2 as a function of frequency between 4 and $24 \mathrm{GHz}$ on November 8, 2017 (black points). The spectrum is clearly inverted between 4 and $15 \mathrm{GHz}$. The red point represents the total flux density at $37 \mathrm{GHz}$ measured by the Metsähovi Radio Observatory quasar monitoring programme. The blue curve shows the best-fit self-absorbed synchrotron spectrum with a fixed optically thick spectral index of +2.5 . See text for more details.

\subsubsection{Magnetic field strength estimated from the synchrotron spectrum}

Core shift is not the only method to estimate the jet magnetic field strength. Since the measured radio spectrum of III Zw 2 in Fig. 6 shows a clear turnover that can be fitted with a selfabsorbed synchrotron spectrum, we can also constrain the jet magnetic field strength by measuring the turnover frequency, $v_{\mathrm{m}}$, the maximum flux density at the turnover, $F_{\mathrm{m}}$, and the size of the emitting region at the turnover frequency, $a\left(v_{\mathrm{m}}\right)$. We fit the spectrum in Fig. 6 with a function describing a self-absorbed synchrotron spectrum emitted by electrons with a power-law energy distribution $N(\gamma)=N_{0} \gamma^{-p}$ in a homogeneous magnetic field (see for instance Pacholczyk 1970):

$F_{v}(v)=F_{\mathrm{m}}\left(\frac{v}{v_{\mathrm{m}}}\right)^{\alpha_{\text {thick }}} \frac{1-\exp \left(-\tau_{\mathrm{m}}\left(v / v_{\mathrm{m}}\right)^{\alpha-\alpha_{\text {thick }}}\right)}{1-\exp \left(-\tau_{\mathrm{m}}\right)}$, where $\tau_{\mathrm{m}}$ is the optical depth at the turnover, $\alpha=-(p-1) / 2$ is the optically thin spectral index, and $\alpha_{\text {thick }}$ is the spectral index of the optically thick part of the spectrum. Following Türler et al. (1999), $\tau_{\mathrm{m}}$ can be approximated as

$\tau_{\mathrm{m}} \approx \frac{3}{2}\left(\sqrt{1-\frac{8 \alpha}{3 \alpha_{\text {thick }}}}-1\right)$.

We fix the optically thick spectral index to $\alpha_{\text {thick }}=+2.5$ in order to approximate a homogeneous emission region. We discuss the consequences of this assumption later.

The best-fitting parameters are $F_{\mathrm{m}}=0.349 \pm 0.030 \mathrm{Jy}$, $v_{\mathrm{m}}=18.3 \pm 1.9 \mathrm{GHz}$, and $\alpha=-0.57 \pm 0.25$. Errors were estimated by fitting 5000 realisations of the spectrum; in each round a set of flux densities were drawn from a set of Gaussian distributions with means corresponding to the measured flux densities and the standard deviations corresponding to the flux density measurement uncertainties at each frequency. We complemented the VLBA flux density measurements with $37 \mathrm{GHz}$ single-dish data from Metsähovi Radio Observatory monitoring programme (Chamani et al. 2020; Teräsranta et al. 1998). Metsähovi observations were made on November 2 and November 16, 2017 and we interpolated the measured flux densities to the date of the VLBA observations, November 8, 2017. Combining data from different angular scales is in this case justified by the point-like core of III Zw 2 and the steep spectrum of its weak kpc-scale emission (the expected contribution of the extended emission is less than $1 \mathrm{mJy}$ at $37 \mathrm{GHz}$; Brunthaler et al. 2005).

Following Marscher (1983), the synchrotron self-absorption magnetic field of a homogeneous, spherical source is

$B_{\mathrm{SSA}}=10^{-5} b(\alpha) a\left(v_{\mathrm{m}}\right)^{4} v_{\mathrm{m}}^{5} F_{\mathrm{m}}^{\prime-2} \frac{\delta}{1+z}[\mathrm{G}]$,

where $a\left(v_{\mathrm{m}}\right)$ is in mas, $v_{\mathrm{m}}$ is in $\mathrm{GHz}, b(\alpha)$ is tabulated in Marscher (1983), and $F_{\mathrm{m}}^{\prime}$ is the flux density at $\nu_{\mathrm{m}}$ in Jy from a linear extrapolation of the optically thin spectral slope. The last value can be expressed in terms of the fitted $F_{\mathrm{m}}$ and $\tau_{\mathrm{m}}$ as

$F_{\mathrm{m}}^{\prime}=\frac{F_{\mathrm{m}} \tau_{\mathrm{m}}}{1-e^{-\tau_{\mathrm{m}}}}$.

In order to use Eq. (16) to calculate the magnetic field strength, we need to resolve the emission region at or near the turnover frequency. However, fitting an elliptical Gaussian to the visibility data results in the minor axis of the Gaussian to go to zero both at 15 and $24 \mathrm{GHz}$. The major axes of these components are oriented at PA of $-50^{\circ}$ and $-56^{\circ}$, respectively. These are relatively close to the orientation of the previous component ejections (Brunthaler et al. 2005; Lister et al. 2019) and we conclude that at our resolution we can only (barely) resolve the jet in the longitudinal direction - not in the transverse direction. Therefore, any estimate of $B_{\mathrm{SSA}}$ is only an upper limit, like our magnetic field estimates from the core-shift analysis.

To estimate an upper limit to $B_{\mathrm{SSA}}$ we fitted both 15 and $24 \mathrm{GHz}$ visibility data with a circular Gaussian and obtained FWHM sizes of $0.066 \pm 0.003$ mas and $0.045 \pm 0.003$ mas, respectively. The uncertainties are estimated following Lampton et al. (1976) like in Sect. 4.1. These size measurements follow the expected $a \propto r \propto v^{-1}$ dependence and we interpolate the Gaussian FWHM size at $v_{\mathrm{m}}$ using this relation. To approximate the size of a partially opaque spherical source - as assumed in Eq. (16) - from a Gaussian FWHM, we approximate $a\left(v_{\mathrm{m}}\right) \approx$ $1.6 \times F W H M$. This corresponds to the size of an optically thick disk for which the visibility amplitude drops to $50 \%$ at the same baseline length as for a Gaussian with the given FWHM. 
Finally, we get $B_{\mathrm{SSA}}=42 \pm 14 \mathrm{mG}$. Since this is an upper limit, we can say that $B_{\mathrm{SSA}} \lesssim 60 \mathrm{mG}(1-\sigma$ upper limit) at the location of the core at $\sim 18 \mathrm{GHz}$. To compare this with $B_{1 \mathrm{pc}}$, we need to estimate an upper limit to the distance of the core at $18 \mathrm{GHz}$ from the jet apex. Following Lobanov (2005) we can estimate that the minimum resolvable size of a Gaussian for our $24 \mathrm{GHz}$ data, which has an on-source $\mathrm{S} / \mathrm{N}$ of 217 , is 0.03 mas. With this upper limit to the transverse width of the jet at $24 \mathrm{GHz}$ and by assuming a conical jet, we can estimate an upper limit to the distance of $18 \mathrm{GHz}$ core from the jet apex, $r(18 \mathrm{GHz}) \lesssim$ $0.4 \mathrm{pc}$. Therefore, we can put a $1-\sigma$ upper limit to $B_{1 \mathrm{pc}} \lesssim 20 \mathrm{mG}$ from our $B_{\mathrm{SSA}}$ upper limit. This is about a factor of three smaller than the upper limit from the core-shift analysis.

If left as a free parameter in the spectral fit, $\alpha_{\text {thick }}=1.58 \pm$ 0.18 , which indicates that the emission region has a non-uniform structure in a sense that the magnetic field and the relativistic particle density have gradients inside it. This is what we also expect from the Blandford-Königl jet model and assume in the coreshift analysis. Therefore, the assumption of a homogeneous selfabsorbed synchrotron source does not, strictly speaking, hold. However, as shown in Marscher (1977), the difference in derived magnetic field strength is modest when $p=2$ and gradients are not overly steep. The ratio $B_{\text {non-uniform }} / B_{\text {uniform }}=0.68$ for $m=1$, $n=3$ and 1.8 for $m=2, n=2$ (see Sect. 3.1. for the definition of the indices $m$ and $n$ ). This does not change our result the upper limit to $B_{1 \mathrm{pc}}$ estimated from the turnover in the synchrotron spectrum is comparable to or smaller than the upper limit estimated from the core-shift limit in equipartition case.

\subsubsection{Limits to the jet magnetic flux}

In order to calculate the jet magnetic flux, $\Phi_{\text {jet}}$, we follow again Zdziarski et al. (2015), who modified the (Zamaninasab et al. 2014) analysis to self-consistently include the observed condition $\Gamma \theta_{\mathrm{j}} \sim 0.1$ and that the observed synchrotron self-absorption magnetic field corresponds to the transverse-average azimuthal magnetic field. From Zdziarski et al. (2015),

$\Phi_{\text {jet }}=\frac{2^{3 / 2} \pi r_{\mathrm{H}} \operatorname{sh} B(1+\sigma)^{1 / 2}}{l a}$,

where the jet magnetisation parameter (ratio of Poynting flux to kinetic energy flux) $\sigma=\left(\Gamma \theta_{\mathrm{j}} / s\right)^{2}, s \lesssim 1$ (Komissarov et al. 2009), $r_{\mathrm{H}}$ represents the $\mathrm{BH}$ event horizon radius, $r_{\mathrm{H}}=$ $r_{g}\left(1+\left(1-a^{2}\right)^{1 / 2}\right), r_{g}=G M_{\mathrm{BH}} / c^{2}$ is the $\mathrm{BH}$ gravitational radius, $h$ is the distance along the jet in parsecs, $l$ is the ratio of the angular frequency of the field lines to the BH angular frequency, and $a$ the BH spin. Setting $l=0.5, s=1$, and $h=1 \mathrm{pc}$, the jet magnetic flux is

$\Phi_{\text {jet }}=8 \times 10^{33} f\left(a_{*}\right)[1+\sigma]^{1 / 2}\left[\frac{M_{\mathrm{BH}}}{10^{9} M_{\odot}}\right]\left[\frac{B}{\mathrm{G}}\right]\left[\mathrm{G} \mathrm{cm}^{2}\right]$,

where

$f(a)=\frac{1}{a} \frac{r_{\mathrm{H}}}{r_{g}}=\frac{1+\left(1-a^{2}\right)^{1 / 2}}{a}$

For $a=1, f(a)=1$ (Zamaninasab et al. 2014), and for III Zw 2 with $a \geq 0.98,1 \leq f(a) \leq 1.2$. We calculated the jet magnetic flux using the core-shift magnetic field strength both with and without equipartition assumption as well as using the SSA magnetic field strength. The upper limit to the magnetic flux in the equipartition case is $\Phi_{\text {jet }}^{\text {eq }} \lesssim 10^{32} \mathrm{G} \mathrm{cm}^{2}$, while the upper limit of the magnetic flux in the non-equipartition case,
$\Phi_{\text {jet }}^{\text {noe }}$, is lower by more than an order of magnitude. The upper limit to the magnetic flux estimated from the SSA spectrum is $\Phi_{\text {jet,SSA }} \lesssim 4 \times 10^{31} \mathrm{G} \mathrm{cm}^{2}$ (see Table 6 ).

Recalling the relation for the predicted magnetic flux threading the $\mathrm{BH}$ (which by flux freezing should be equal $\Phi_{\text {jet }}$ ) in the MAD state (Narayan et al. 2003; Tchekhovskoy et al. 2011),

$\Phi_{\mathrm{BH}, \mathrm{MAD}}=50\left(\dot{M} r_{g}^{2} c\right)^{1 / 2}$,

which is equivalent to

$$
\begin{aligned}
\Phi_{\mathrm{BH}, \mathrm{MAD}}= & 2.4 \times 10^{34}\left[\frac{\eta}{0.4}\right]^{-1 / 2}\left[\frac{M_{\mathrm{BH}}}{10^{9} M_{\odot}}\right] \\
& \times\left[\frac{L_{\mathrm{acc}}}{1.26 \times 10^{47} \mathrm{erg} \mathrm{s}^{-1}}\right]^{1 / 2}\left[\mathrm{G} \mathrm{cm}^{2}\right],
\end{aligned}
$$

where $\eta$ is the radiative efficiency of the accretion disk. We note that the higher $L_{\text {acc }}$ for III Zw 2 from Falcke et al. (1995) is the luminosity of the UV-bump derived by spectral fitting, while the lower $L_{\text {acc }}$ from Berton et al. (2015) is derived by a scaling relation from the luminosity of the $\mathrm{H} \beta$ line (see Table 5).

By applying Eq. (22) and assuming $\eta=0.4$ as in Zamaninasab et al. (2014), we find that the upper limit of $\Phi_{\text {jet }}^{\text {eq }}$ for III Zw 2 is smaller by a factor of five with respect to the predicted

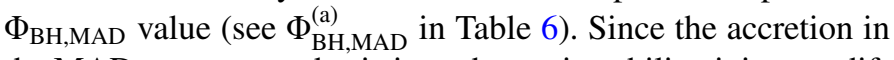
the MAD state proceeds via interchange instability, it is very difficult to know the actual radiative efficiency of a MAD. However, based on the discussion in Zdziarski et al. (2015) and on the relatively low Eddington ratio of III Zw 2 (0.04-0.1; Berton et al. 2015; Inoue et al. 2007), we argue here that the radiative efficiency is likely smaller than 0.4 for III Zw 2. For instance, Davis \& Laor (2011) derived the radiative efficiency for a sample of PG quasars (including III Zw 2) by employing optical spectroscopy measurements assuming a standard thin accretion disk that gives $\eta=0.19$, and from $\mathrm{BH}$ mass and stellar velocity dispersion relationship, resulting in $\eta=0.13$. By adopting these efficiencies although not directly applicable in a MAD case - the upper limit to the jet magnetic flux is smaller than $\Phi_{\mathrm{BH}, \mathrm{MAD}}$ by a factor of seven and nine respectively (for comparisons, see (b) $\Phi_{\mathrm{BH}, \mathrm{MAD}}$ in Table 6). Since the $L_{\text {acc }}$ from Falcke et al. (1995) is likely to be more accurate, we also calculate $\Phi_{\mathrm{BH}, \mathrm{MAD}}$ using that value only (see $\Phi_{\mathrm{BH}, \mathrm{MAD}}^{(\mathrm{c})}$ in Table 6). This results in more than an order of magnitude higher value than our upper limit to $\Phi_{\text {jet }}^{\text {eq }}$.

\section{Summary and discussion}

Active galactic nuclei show a wide range of radio loudness that can be considered as a proxy for their jet production efficiency. It is still unclear which mechanisms are responsible for this variety even among sources with otherwise similar properties. One explanation is that the spread in the $\mathrm{BH}$ spin alone determines the range of observed radio loudness. Another possible controlling parameter of the radio loudness is the magnetic flux threading the $\mathrm{BH}$. In this so-called magnetic flux paradigm, it is the accretion history of the AGN that determines how much magnetic flux has accumulated in the inner parts of the accretion disk and only those sources that have developed a MAD can have high jet production efficiency and consequently radio loudness (Sikora \& Begelman 2013; Xie \& Zdziarski 2019). This model was successfully tested with a powerful radio-loud AGN sample that showed jet magnetic flux values consistent with the prediction of the MAD scenario (Zamaninasab et al. 2014). 


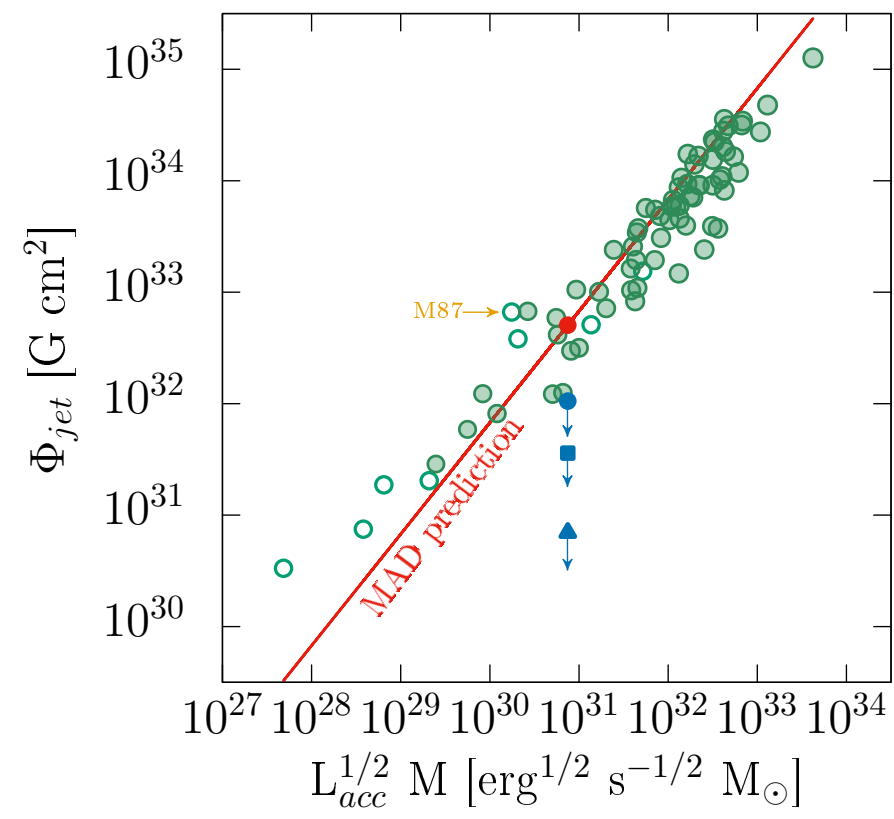

Fig. 7. Plot adapted from Zamaninasab et al. (2014). In their work, they assumed $\Gamma \theta_{\mathrm{j}}=1$ for the whole sample. The open and filled green circles represent our corrected values with $\Gamma \theta_{\mathrm{j}} \neq 1$ for radio galaxies and $\Gamma \theta_{\mathrm{j}}=0.13$ for blazars, respectively. The red and blue filled-circles represent the MAD predicted and measured (upper limit in equipartition) values of the jet magnetic flux of III Zw 2. The blue triangle represents the measured upper limit without equipartition assumption. The blue square represents the measured upper limit from the synchrotron selfabsorption magnetic field $B_{\mathrm{SSA}}$.

However, the model has not yet been tested with low radioloudness sources, which would be expected to show lower jet magnetic fluxes - well below the MAD level - for similar BH spins.

In this work, we tested the magnetic flux paradigm with the RIQ IIIZw 2. First, we analysed multi-frequency phasereferencing VLBA data and obtained an upper limit for the core shift. This allowed us to put upper limits to the jet magnetic field strength and the magnetic flux both with and without assuming equipartition conditions in the jet. We found that III Zw 2 has not reached the MAD level magnetic flux in either scenario. By assuming equipartition, the magnetic flux is less than $20 \%$ of the MAD level. The failure to reach the MAD state is reinforced under the non-equipartition scenario where the magnetic flux is below the MAD limit by more than an order of magnitude. Another supporting result comes from the fitting of the synchrotron spectrum, which leads to an upper limit of the magnetic flux below the MAD limit by one order of magnitude. The values are displayed in Fig. 7 where the observed and the predicted MAD magnetic fluxes are plotted in the figure adapted from Zamaninasab et al. (2014) ${ }^{2}$ These results can naturally explain the relatively low jet production efficiency in this RIQ despite it hosting a fast spinning SMBH. The results are in good agreement with the magnetic flux paradigm.

\footnotetext{
2 We corrected $B_{1 \mathrm{pc}}^{\prime}$ in their whole sample by multiplying it by a factor of $(1+z)^{1 / 4}$, which is missing in their original work (see Zdziarski et al. 2015). The magnetic fluxes are estimated with Eq. (19), and they are slightly lower than the values presented in Zamaninasab et al. (2014) due to the difference in the value of $\Gamma \theta_{\mathrm{j}}$.
}

Another way to look at this is the notion by Nalewajko et al. (2014) that the $\Phi_{\text {jet }} \sim \Phi_{\mathrm{BH}, \mathrm{MAD}}$ condition expected in the MAD case is equivalent to the condition that the jet magnetic power, $L_{B}=7.14 \times 10^{46}\left(\Gamma \theta_{\mathrm{j}}\right)^{2} B_{1 \mathrm{pc}}^{\prime 2}\left[\mathrm{erg} \mathrm{s}^{-1}\right]$, is comparable to the accretion disk luminosity, $L_{\mathrm{acc}}$. In the case of III Zw $2, L_{B} \lesssim$ $10^{43} \mathrm{erg} \mathrm{s}^{-1}$ (for $\theta_{\mathrm{j}}=6.7^{\circ}$ ), which is two orders of magnitude lower than the mean disk luminosity of $1.7 \times 10^{45} \mathrm{erg} \mathrm{s}^{-1}$. This calculation consistently shows that the source has not reached the MAD state, which, however, is not surprising considering that it uses the same $B_{1 \mathrm{pc}}^{\prime}, \Gamma \theta_{\mathrm{j}}$ and $L_{\mathrm{acc}}$ as the magnetic flux calculations.

The order-of-magnitude discrepancy between the upper limits to $B_{1 \mathrm{pc}}^{\text {noeq }}$ and $B_{1 \mathrm{pc}}^{\mathrm{eq}}$ requires some attention. Since the ratio $B_{1 \mathrm{pc}}^{\mathrm{eq}} / B_{1 \mathrm{pc}}^{\text {noe }}$ scales as $\Delta r^{-17 / 4}$, the discrepancy only becomes larger if the true core-shift value is less than our upper limit. Therefore, we have a lower limit to the ratio $B_{1 \mathrm{pc}}^{\mathrm{eq}} / B_{1 \mathrm{pc}}^{\text {noeq }}$, which would in principle indicate that the VLBI core in III Zw 2 is particle energy dominated by a quite large factor of $\gtrsim 5 \times 10^{4}$. However, we note that $B_{1 \mathrm{pc}}^{\text {noeq }}$ is very sensitive to the measured quantities. Confirming the possible deviation from the equipartition will therefore require an accurate measurement of the core shift instead of the upper limit that we have now.

The magnetic flux paradigm appears to explain the RIQlevel jet production efficiency in III Zw 2; thus, we discuss below some possibilities for the low accumulated magnetic flux in this source:

- Sikora \& Begelman (2013) suggest that RIQ sources could be linked to a stochastic jet production triggered by magnetic field fluctuations due to turbulence in the inner hot or geometrically thick region of the accretion flow. If the fluctuations have large enough spatial coherence length and strength, they could deposit enough magnetic flux to the $\mathrm{BH}$ to produce an intermittent jet. There is some evidence that the jet in III Zw 2 could be of intermittent nature. First, the source exhibits highly fluctuating radio flux density at $37 \mathrm{GHz}$ (more than a factor of ten peak-to-through ratio; Chamani et al. 2020), which could be due to variable jet power. It undergoes a recurrent activity roughly every five years ( $\mathrm{Li}$ et al. 2010) with a detected variable gamma-ray emission in the short term (Liao et al. 2016). Secondly, the radio morphology is point-like most of the time, but occasionally a blob is ejected (Brunthaler et al. 2005; Lister et al. 2019).

- Early morphology studies indicated that III Zw 2 is hosted in a spiral galaxy (Hutchings \& Campbell 1983; Taylor et al. 1996). If the host galaxy of III Zw 2 indeed is a disk, this may be connected to the failure of the development of a powerful jet. After all, radio-loud AGN are typically associated with elliptical galaxies - although there are exceptions, such as jetted narrow line Seyfert 1 galaxies (Lähteenmäki et al. 2018). Blandford et al. (2019) speculate that the mostly equatorial large-scale gas inflow towards the central region in spiral galaxies could lead to difficulties in accumulating and trapping magnetic flux within the $\mathrm{BH}$ radius of influence. In such an equatorial flow, field lines become radial on the surface of the disk and may easily dissipate through reconnection. However, the gas inflow in spiral galaxies may not stay equatorial from the kiloparsec scales all the way down to the $\mathrm{BH}$ given the complex nuclear structures (see for instance Hopkins \& Quataert 2010, 2011; Combes et al. 2014; Chamani et al. 2017; Pjanka et al. 2017). It is therefore unclear if a difficulty in transporting large-scale magnetic 
fields from the galactic scale to the central $\lesssim \mathrm{kpc}$ is enough to hinder the build-up of the magnetic flux in the $\mathrm{BH}$ sphere of influence.

- Later observations with the Hubble Space Telescope reported by Veilleux et al. (2009) suggest that III Zw 2 resides in an elliptical galaxy instead. In such a case, magnetic flux could have been supplied from the large scales in a short hot accretion phase, which - for some reason - has not been long enough to build up enough magnetic flux to create a MAD - or perhaps the accreted field was too disordered. As long as the host galaxy morphology is unclear, it is difficult to address these questions. Interestingly, it appears that III Zw 2 is in an on-going merger (Surace et al. 2001; Veilleux et al. 2009). Since the interaction can efficiently drive gas into the inner region, the merger may have triggered the on-going cold mode accretion phase of the AGN in III Zw 2. As a sidenote, Sikora \& Begelman (2013) propose that a merger of two disk galaxies where there has never been an extended hot accretion phase - and therefore little accumulation of magnetic flux - represents one possible evolutionary track to a RQQ.

The core shift in III Zw 2 is below our measurement accuracy and we note that this is likely partly due to our calibrators. The multi-frequency images of III Zw 2 show that the source is point-like with no strong or extended jet structure. Unfortunately, this is not true for the calibrators and our measurement accuracy of the core shift of III Zw 2 was degraded by all calibrators being extended sources that exhibit significant core shifts (up to 0.77 mas between 4 and $24 \mathrm{GHz}$ ) themselves. Removing these calibrator core shifts introduces additional uncertainties as demonstrated in Sect. 4.1. In the case of the calibrator J0007, the jet exhibits a significant bend, which introduces further uncertainty on the resulting core-shift values with our method. Another source of uncertainty has to do with the proper core-identification in phase-referenced images, especially in the $C$-band.

We conclude by noting that we have here and in Chamani et al. (2020) demonstrated a new method for testing the magnetic flux paradigm by combining BH spin measurements from $\mathrm{X}$-ray reflection spectroscopy with jet magnetic field measurements from sub-milliarcsecond multi-frequency VLBI astrometry. We have shown for one particular RIQ source that its measured $\mathrm{BH}$ spin and jet magnetic flux are in a good agreement with the Sikora \& Begelman (2013) model, in which the amount of magnetic flux threading the $\mathrm{BH}$ is the parameter controlling the AGN jet production efficiency. Obviously, we have tested our method only for one source and these studies should be extended to a larger sample of radio-intermediate and radioquiet sources in order to cover a range of radio loudness and Eddington ratios.

Acknowledgements. The authors thank Karri Koljonen for his help in the preparation of the observing proposals, Maria Rioja, Richard Dodson, Andreas Brunthaler and Andrzej Zdziarski for helpful discussions, and Eduardo Ros for careful reading of the manuscript. This work was supported partly by the Academy of Finland projects 274477 and 315721 . W. Chamani is thankful for the visiting joint research grant supported by the National Astronomical Observatory of Japan (NAOJ). The Very Long Baseline Array (VLBA) is an instrument of the National Radio Astronomy Observatory. The National Radio Astronomy Observatory is a facility of the National Science Foundation operated under cooperative agreement by Associated Universities, Inc. This work made use of the Swinburne University of Technology software correlator, developed as part of the Australian Major National Research Facilities Programme and operated under licence. This publication makes use of data obtained at the Metsähovi Radio Observatory, operated by the Aalto University in Finland.

\section{References}

Berton, M., Foschini, L., Ciroi, S., et al. 2015, A\&A, 578, A28 Blandford, R. D., \& Königl, A. 1979, ApJ, 232, 34

Blandford, R. D., \& Znajek, R. L. 1977, MNRAS, 179, 433

Blandford, R., Meier, D., \& Readhead, A. 2019, ARA\&A, 57, 467

Blundell, K. M., \& Beasley, A. J. 1998, MNRAS, 299, 165

Brunthaler, A., Falcke, H., Bower, G. C., et al. 2000, A\&A, 357, L45

Brunthaler, A., Falcke, H., Bower, G. C., et al. 2005, A\&A, 435, 497

Chamani, W., Dörschner, S., \& Schleicher, D. R. G. 2017, A\&A, 602, A84

Chamani, W., Koljonen, K., \& Savolainen, T. 2020, A\&A, 635, A172

Clausen-Brown, E., Savolainen, T., Pushkarev, A. B., Kovalev, Y. Y., \& Zensus, J. A. 2013, A\&A, 558, A144

Combes, F., García-Burillo, S., Casasola, V., et al. 2014, A\&A, 565, A97

Cooper, N. J., Lister, M. L., \& Kochanczyk, M. D. 2007, ApJS, 171, 376

Croke, S. M., \& Gabuzda, D. C. 2008, MNRAS, 386, 619

Davis, S. W., \& Laor, A. 2011, ApJ, 728, 98

Dodson, R., Rioja, M. J., Molina, S. N., \& Gómez, J. L. 2017, ApJ, 834, 177

Falcke, H., Malkan, M. A., \& Biermann, P. L. 1995, A\&A, 298, 375

Falcke, H., Patnaik, A. R., \& Sherwood, W. 1996a, ApJ, 473, L13

Falcke, H., Sherwood, W., \& Patnaik, A. R. 1996b, ApJ, 471, 106

Fromm, C. M., Ros, E., Perucho, M., et al. 2013, A\&A, 557, A105

Ghisellini, G., Haardt, F., \& Matt, G. 2004, A\&A, 413, 535

Ghisellini, G., Tavecchio, F., Maraschi, L., Celotti, A., \& Sbarrato, T. 2014, Nature, 515, 376

Greisen, E. W. 2003, AIPS, the VLA, and the VLBA (Kluwer Academic Publishers), 285, 109

Grier, C. J., Peterson, B. M., Pogge, R. W., et al. 2012, ApJ, 755, 60

Hada, K., Doi, A., Kino, M., et al. 2011, Nature, 477, 185

Haga, T., Doi, A., Murata, Y., et al. 2015, ApJ, 807, 15

Hernán Caballero, A. 2012, MNRAS, 427, 816

Hirotani, K. 2005, ApJ, 619, 73

Hodgson, J. A., Krichbaum, T. P., Marscher, A. P., et al. 2017, A\&A, 597, A80

Hopkins, P. F., \& Quataert, E. 2010, MNRAS, 407, 1529

Hopkins, P. F., \& Quataert, E. 2011, MNRAS, 415, 1027

Hovatta, T., \& Lindfors, E. 2019, New A Rev., 87

Hovatta, T., Valtaoja, E., Tornikoski, M., \& Lähteenmäki, A. 2009, A\&A, 494, 527

Hutchings, J. B., \& Campbell, B. 1983, Nature, 303, 584

Inoue, H., Terashima, Y., \& Ho, L. C. 2007, ApJ, 662, 860

Kellermann, K. I., Sramek, R., Schmidt, M., Shaffer, D. B., \& Green, R. 1989, AJ, 98, 1195

Komissarov, S. S., Vlahakis, N., Königl, A., \& Barkov, M. V. 2009, MNRAS, 394, 1182

Kovalev, Y. Y., Lobanov, A. P., Pushkarev, A. B., \& Zensus, J. A. 2008, A\&A, 483, 759

Kutkin, A. M., Sokolovsky, K. V., Lisakov, M. M., et al. 2014, MNRAS, 437, 3396

Lähteenmäki, A., Järvelä, E., Ramakrishnan, V., et al. 2018, A\&A, 614, L1

Lampton, M., Margon, B., \& Bowyer, S. 1976, ApJ, 208, 177

Li, H., Xie, G., Dai, H., et al. 2010, New Astron., 15, 254

Liao, N.-H., Xin, Y.-L., Fan, X.-L., et al. 2016, ApJS, 226, 17

Lister, M. L., Homan, D. C., Hovatta, T., et al. 2019, ApJ, 874, 43

Lobanov, A. P. 1998, A\&A, 330, 79

Lobanov, A. P. 2005, ArXiv e-prints [arXiv: 0503225]

Marscher, A. P. 1977, ApJ, 216, 244

Marscher, A. P. 1983, ApJ, 264, 296

McKinney, J. C., Tchekhovskoy, A., \& Blandford, R. D. 2012, MNRAS, 423, 3083

McNamara, B. R., Rohanizadegan, M., \& Nulsen, P. E. J. 2011, ApJ, 727, 39

Mioduszewski, A. J., \& Kogan, L. 2009, Strategy for Removing Tropospheric and Clock Errors Using DELZN. AIPS Memo 110 (NRAO)

Moderski, R., Sikora, M., \& Lasota, J.-P. 1998, MNRAS, 301, 142

Nalewajko, K., Sikora, M., \& Begelman, M. C. 2014, ApJ, 796, L5

Narayan, R., Igumenshchev, I. V., \& Abramowicz, M. A. 2003, PASJ, 55, L69

Nyland, K., Dong, D. Z., Patil, P., et al. 2020, ApJ, 905, 74

Orosz, G., Imai, H., Dodson, R., et al. 2017, AJ, 153, 119

O'Sullivan, S. P., \& Gabuzda, D. C. 2009, MNRAS, 400, 26

Pacholczyk, A. G. 1970, Radio Astrophysics. Nonthermal Processes in Galactic and Extragalactic Sources

Pjanka, P., Greene, J. E., Seth, A. C., et al. 2017, ApJ, 844, 165

Plavin, A. V., Kovalev, Y. Y., Pushkarev, A. B., \& Lobanov, A. P. 2019, MNRAS, 485, 1822

Pushkarev, A. B., Hovatta, T., Kovalev, Y. Y., et al. 2012, A\&A, 545, A113

Pushkarev, A. B., Kovalev, Y. Y., Lister, M. L., \& Savolainen, T. 2017, MNRAS, 468, 4992

Pushkarev, A. B., Butuzova, M. S., Kovalev, Y. Y., \& Hovatta, T. 2018, MNRAS, 482, 2336 
Raginski, I., \& Laor, A. 2016, MNRAS, 459, 2082

Rawlings, S., \& Saunders, R. 1991, Nature, 349, 138

Reid, M. J., \& Honma, M. 2014, ARA\&A, 52, 339

Reid, M. J., Menten, K. M., Brunthaler, A., et al. 2009, ApJ, 693, 397

Reynolds, C. 2021, in 43rd COSPAR Scientific Assembly. Held 28 January - 4 February, 43, 1412

Reynolds, C. S. 2013, Space Sci. Rev., 183, 277

Savolainen, T., Wiik, K., Valtaoja, E., \& Tornikoski, M. 2008, ASP Conf. Ser. 386,451

Shepherd, M. C. 1997, ASP Conf. Ser., 125, 77

Sikora, M., \& Begelman, M. C. 2013, ApJ, 764, L24

Sikora, M., Stawarz, Ł., \& Lasota, J.-P. 2007, ApJ, 658, 815

Snellen, I. A. G., McMahon, R. G., Hook, I. M., \& Browne, I. W. A. 2002, MNRAS, 329, 700

Sokolovsky, K. V., Kovalev, Y. Y., Pushkarev, A. B., \& Lobanov, A. P. 2011, A\&A, 532, A38

Strittmatter, P. A., Hill, P., Pauliny-Toth, I. I. K., Steppe, H., \& Witzel, A. 1980, A\&A, 88, L12

Surace, J. A., Sanders, D. B., \& Evans, A. S. 2001, AJ, 122, 2791

Taylor, G. L., Dunlop, J. S., Hughes, D. H., \& Robson, E. I. 1996, MNRAS, 283, 930

Tchekhovskoy, A., Narayan, R., \& McKinney, J. C. 2010, ApJ, 711, 50
Tchekhovskoy, A., Narayan, R., \& McKinney, J. C. 2011, MNRAS, 418, L79

Teräsranta, H., Tornikoski, M., Mujunen, A., et al. 1998, A\&AS, 132, 305

Thompson, A. R., Moran, J. M., \& Swenson, G. W., J. 2017, Interferometry and Synthesis in Radio Astronomy, 3rd Edn.. (Springer)

Türler, M., Courvoisier, T. J. L., \& Paltani, S. 1999, A\&A, 349, 45

Unger, S. W., Lawrence, A., Wilson, A. S., Elvis, M., \& Wright, A. E. 1987, MNRAS, 228, 521

Veilleux, S., Kim, D. C., Rupke, D. S. N., et al. 2009, ApJ, 701, 587

Voitsik, P. A., Pushkarev, A. B., Kovalev, Y. Y., et al. 2018, Astron. Rep., 62, 787

Volonteri, M., Sikora, M., Lasota, J. P., \& Merloni, A. 2013, ApJ, 775, 94

Walker, R. C., Dhawan, V., Romney, J. D., Kellermann, K. I., \& Vermeulen, R. C. 2000, ApJ, 530, 233

White, S. V., Jarvis, M. J., Kalfountzou, E., et al. 2017, MNRAS, 468, 217

Wright, E. L. 2006, PASP, 118, 1711

Xie, F.-G., \& Zdziarski, A. A. 2019, ApJ, 887, 167

Zakamska, N. L., \& Greene, J. E. 2014, MNRAS, 442, 784

Zamaninasab, M., Clausen-Brown, E., Savolainen, T., \& Tchekhovskoy, A. 2014, Nature, 510, 126

Zdziarski, A. A., Sikora, M., Pjanka, P., \& Tchekhovskoy, A. 2015, MNRAS, 451,927 


\section{Appendix A: Phase-referenced images}

Phase-referenced images of the calibrator sources at the four frequencies displayed below in Figs. A.1-A.3.

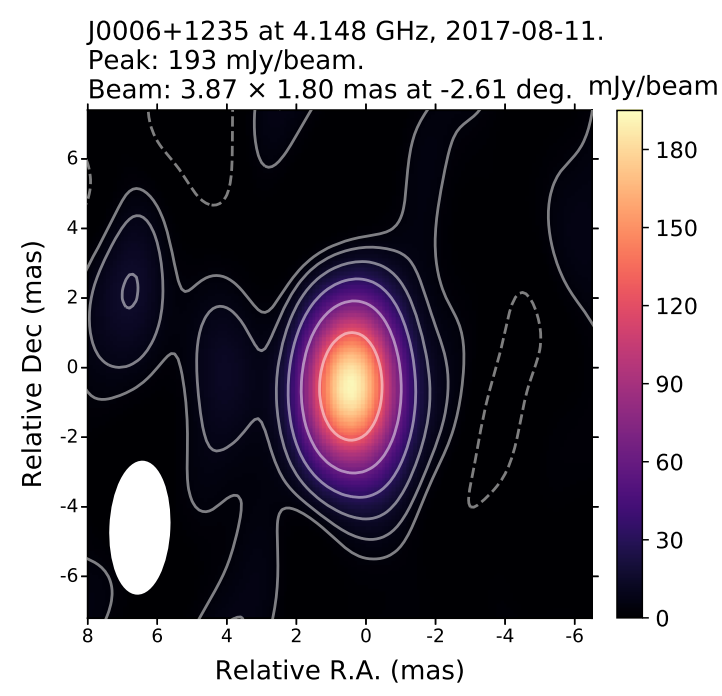

(a)

J0006+1235 at $15.352 \mathrm{GHz}, 2017-08-11$. Peak: $140 \mathrm{mJy} / \mathrm{beam}$.

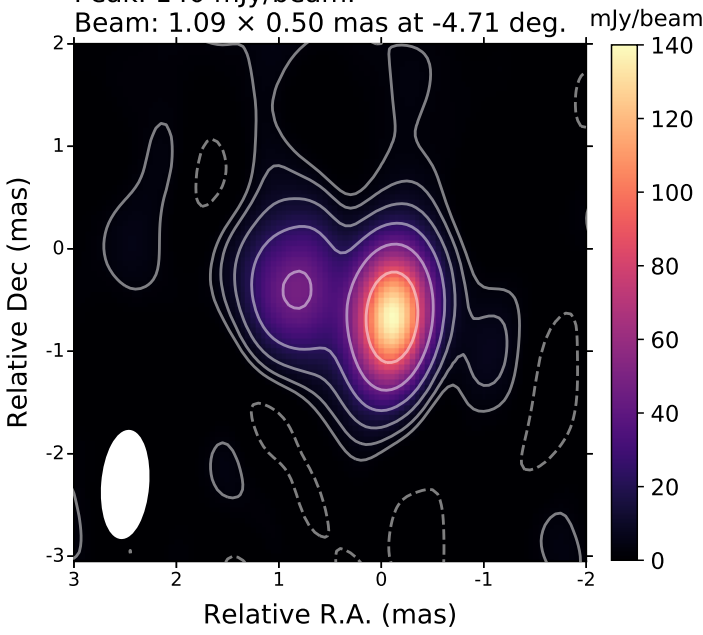

(c)

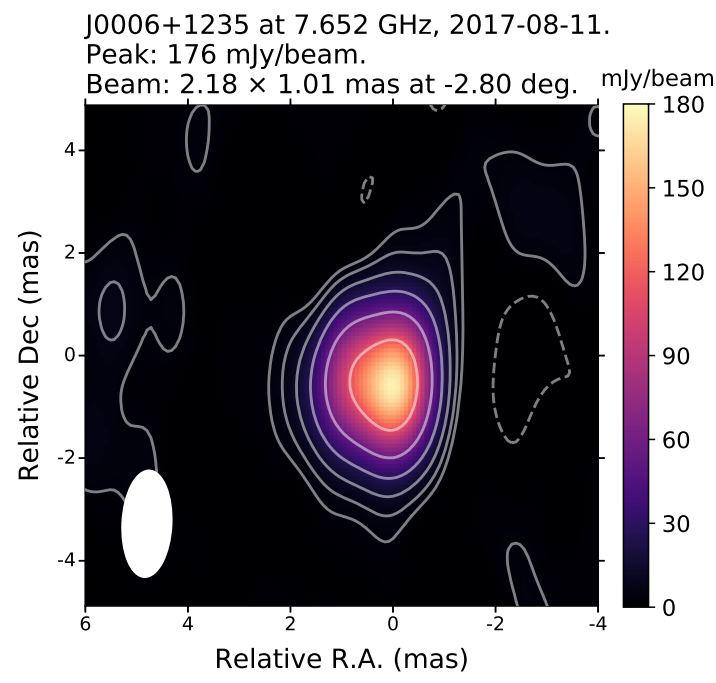

(b)

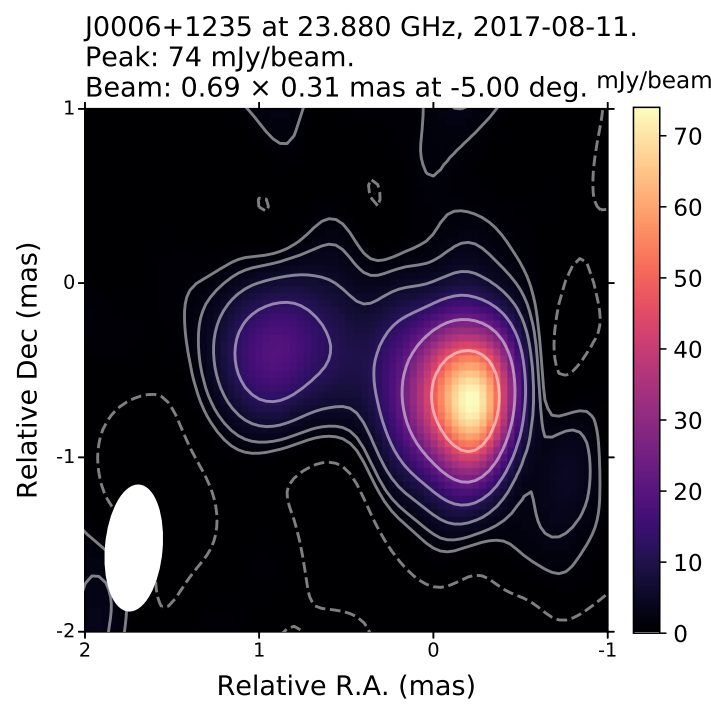

(d)

Fig. A.1. Phase-referenced clean images of J0006+1235. The images were obtained at (a) $4.148 \mathrm{GHz}$, (b) $7.652 \mathrm{GHz}$, (c) $15.352 \mathrm{GHz}$ and (d) 23.88 GHz. The rms noise level from the lower to the higher frequency is $2.6,2,1.7,1.3 \mathrm{mJy}_{\text {beam }}^{-1}$. The interferometric beam (ellipse) is displayed on the bottom-left corner of each image. Contours represent $-2 \%, 2 \%, 4 \%, 8 \%, 16 \%, 32 \%$, and $64 \%$ of the peak intensity at each image. 
W. Chamani et al.: Testing the magnetic flux paradigm for radio loudness of AGN

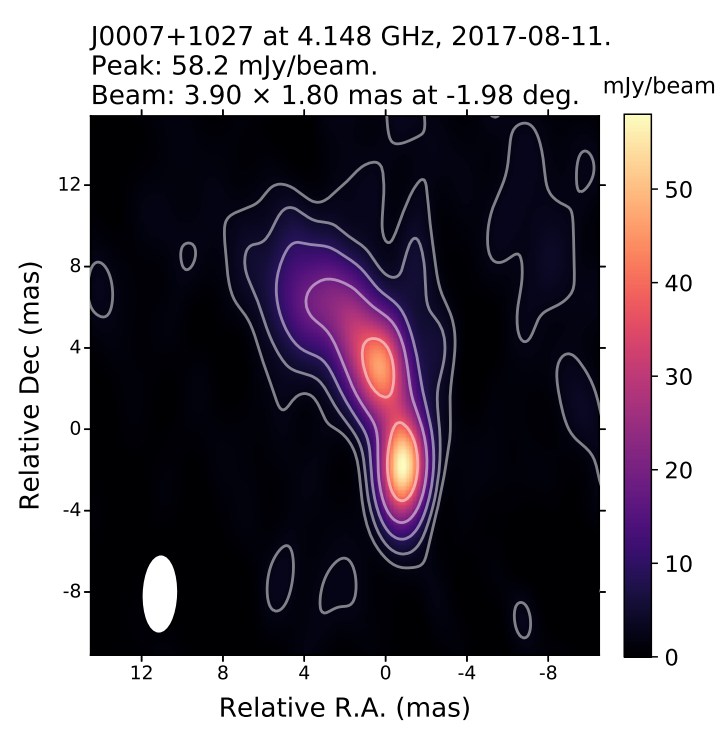

(a)

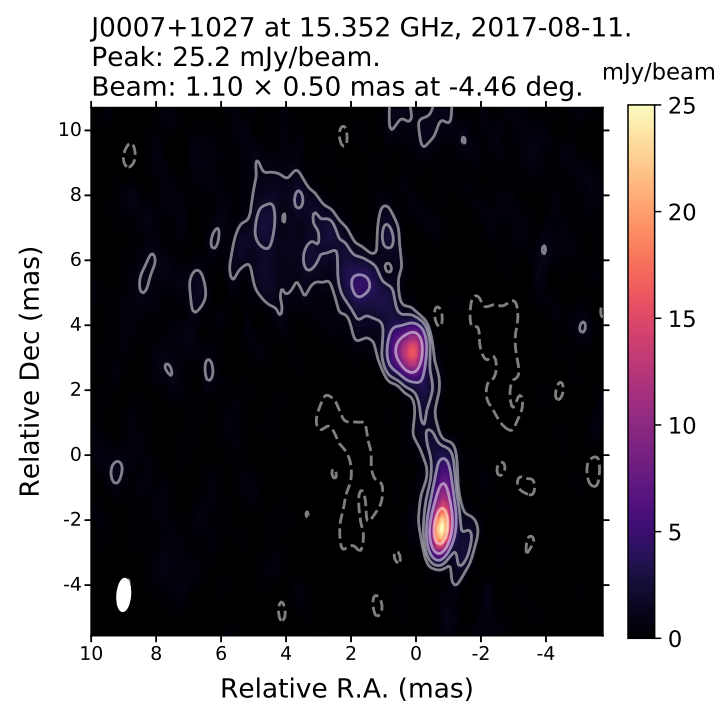

(c)

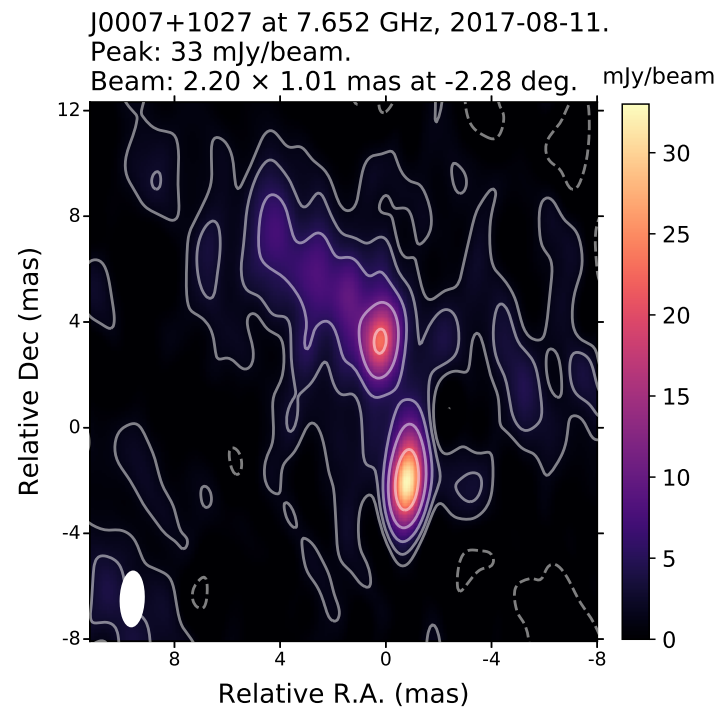

(b)

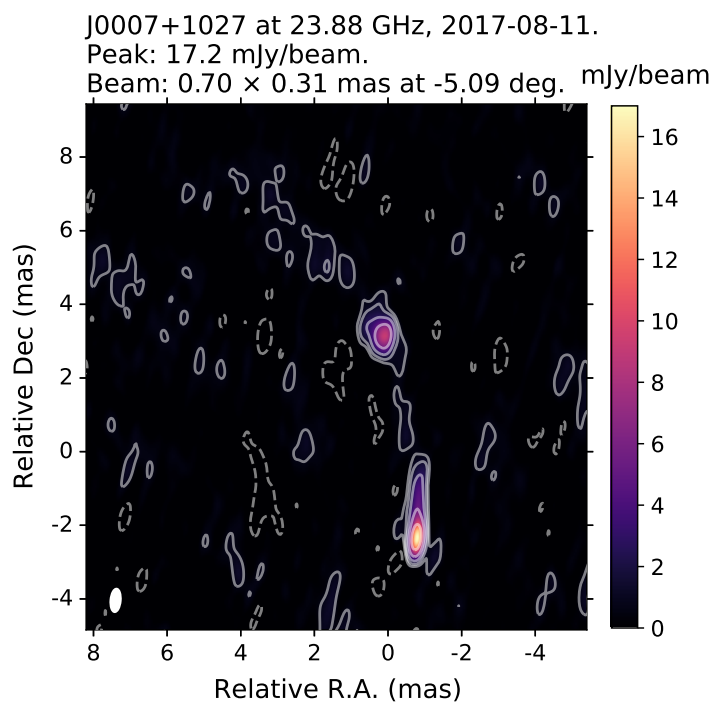

(d)

Fig. A.2. Phase-referenced clean images of J0007+1027. The images were obtained at (a) $4.148 \mathrm{GHz}$, (b) $7.652 \mathrm{GHz}$, (c) $15.352 \mathrm{GHz}$ and (d) $23.88 \mathrm{GHz}$. The rms noise level from the lower to the higher frequency is $1,1.2,0.5,0.3 \mathrm{mJy}^{-1}$ beam $^{-1}$. The interferometric beam (ellipse) is displayed on the bottom-left corner of each image. Contours represent $-4 \%, 4 \%, 8 \%, 16 \%, 32 \%$, and $64 \%$ of the peak intensity at each image. 


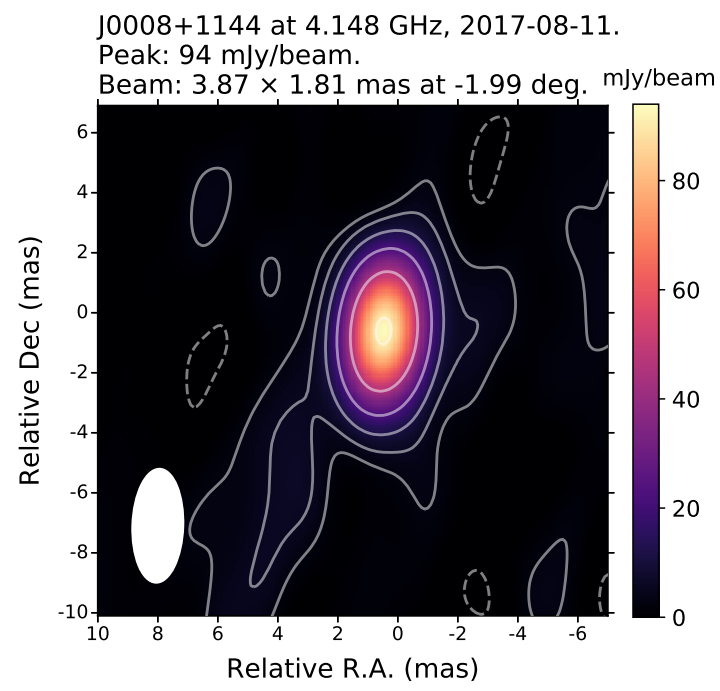

(a)

J0008+1144 at $15.352 \mathrm{GHz}, 2017-08-11$. Peak: $42.3 \mathrm{mJy} / \mathrm{beam}$.

Beam: $1.09 \times 0.50$ mas at $-4.21 \mathrm{deg} . \mathrm{mJy} / \mathrm{beam}$

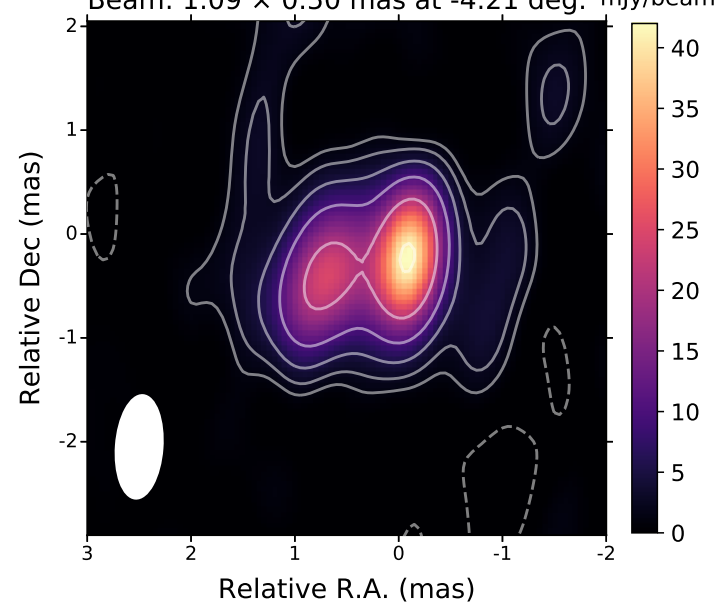

(c)

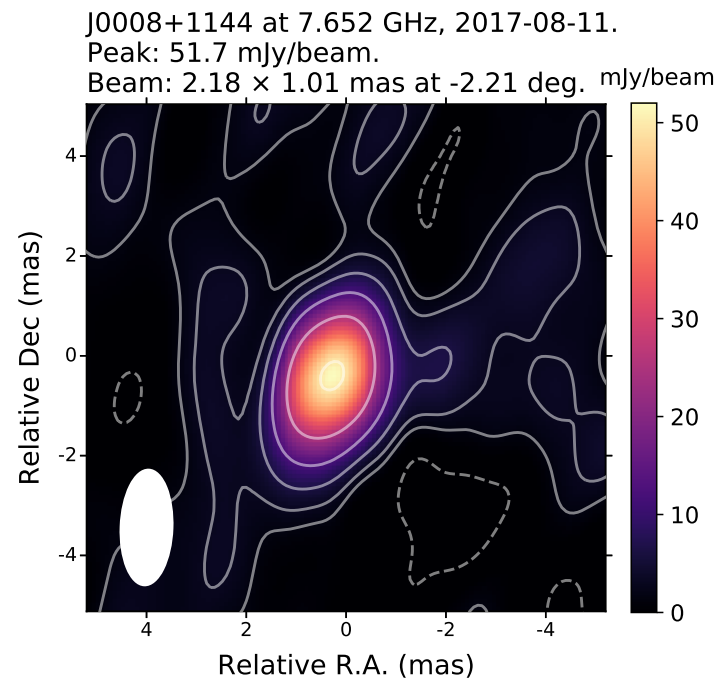

(b)

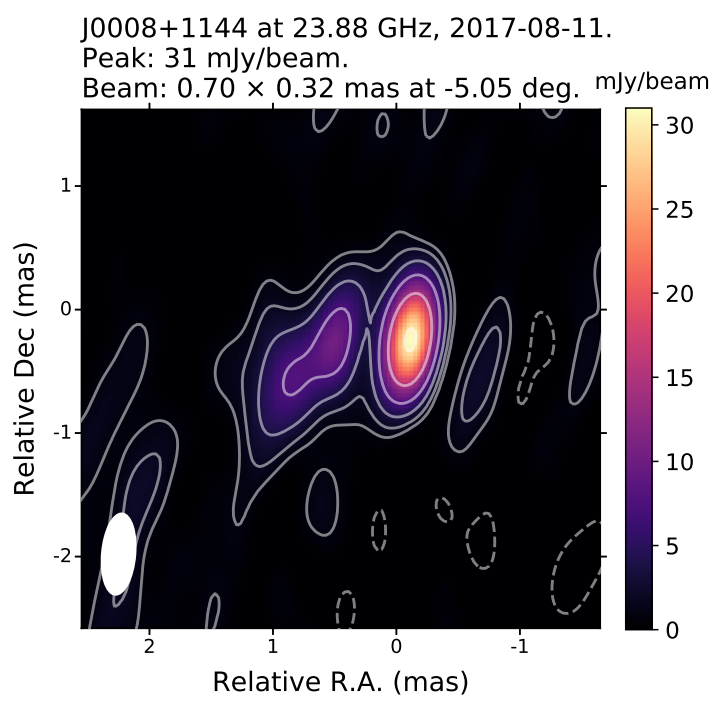

(d)

Fig. A.3. Phase-referenced clean images of J0008+1144. The images were obtained at (a) $4.148 \mathrm{GHz}$, (b) $7.652 \mathrm{GHz}$, (c) $15.352 \mathrm{GHz}$ and (d) $23.88 \mathrm{GHz}$. The rms noise level from the lower to the higher frequency is $1.1,1.4,0.7,0.5 \mathrm{mJy} \mathrm{beam}^{-1}$. The interferometric beam (ellipse) is displayed on the bottom-left corner of each image. Contours represent $-2 \%, 2 \%, 4 \%, 8 \%, 16 \%, 32 \%$, and $64 \%$ of the peak intensity at each image. 
W. Chamani et al.: Testing the magnetic flux paradigm for radio loudness of AGN

\section{Appendix B: Self-calibrated images}

The CLEAN images of the calibrator sources after selfcalibration in amplitudes and in phases are displayed below in Figs. B.1-B.3.

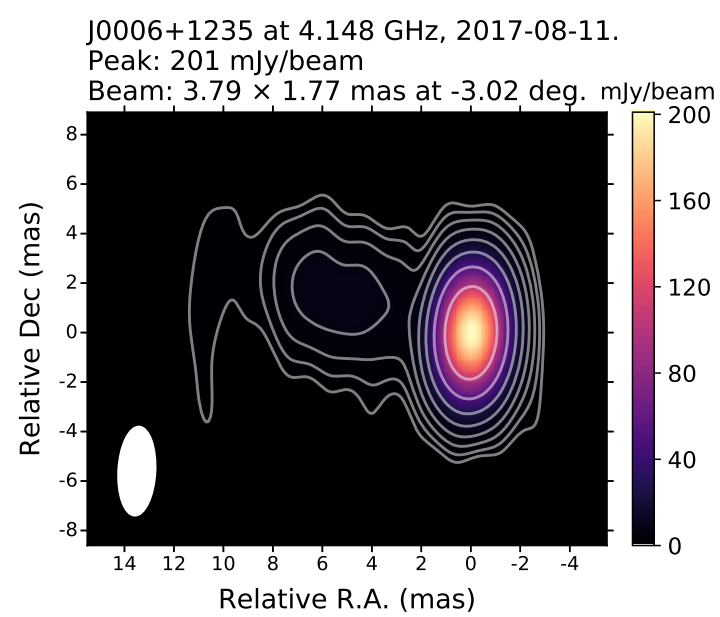

(a)

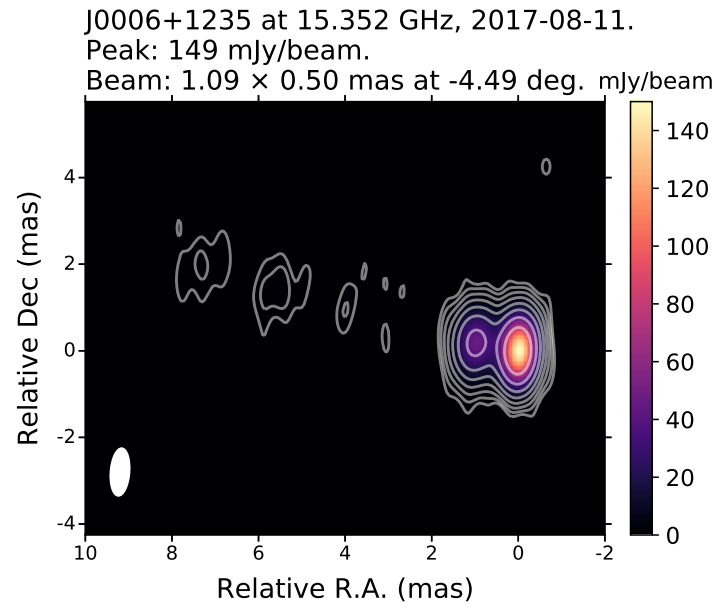

(c)

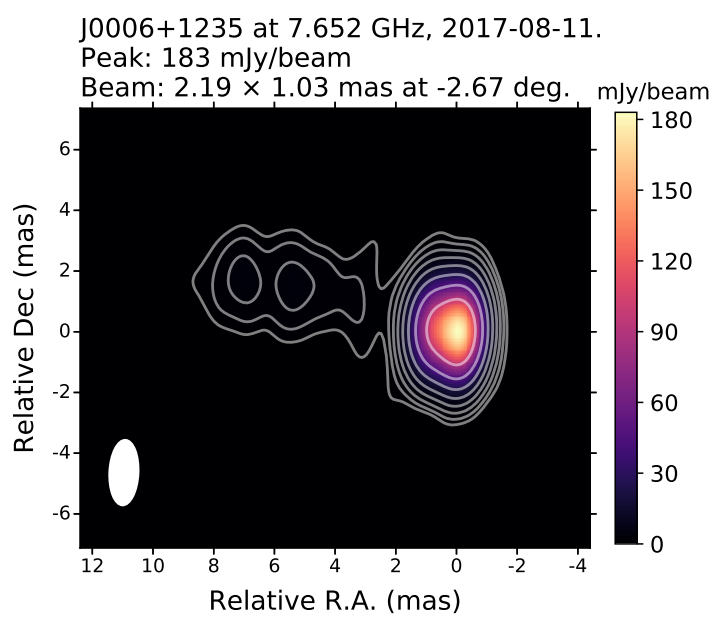

(b)

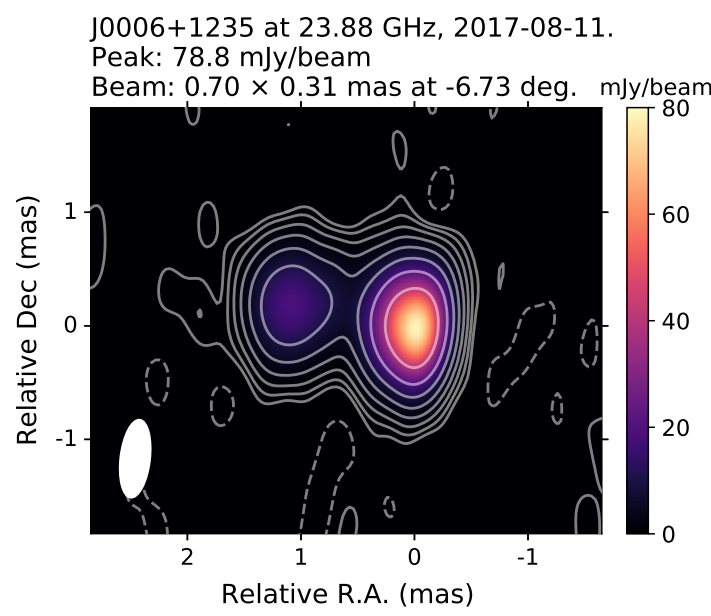

(d)

Fig. B.1. Self-calibrated images of J0006+1235. The images were obtained at (a) $4.148 \mathrm{GHz}$, (b) $7.652 \mathrm{GHz}$, (c) $15.352 \mathrm{GHz}$ and (d) $23.88 \mathrm{GHz}$. The rms noise level from the lower to the higher frequency is $0.3,0.1,0.2,0.2$ mJy beam $^{-1}$. The interferometric beam (ellipse) is displayed on the bottom-left corner of each image. Contours represent $-0.4 \%, 0.4 \%, 0.8 \%, 1.6 \%, 3.2 \%, 6.4 \%, 12.8 \%, 25.6 \%$, and $51.2 \%$ of the peak intensity at each image. 


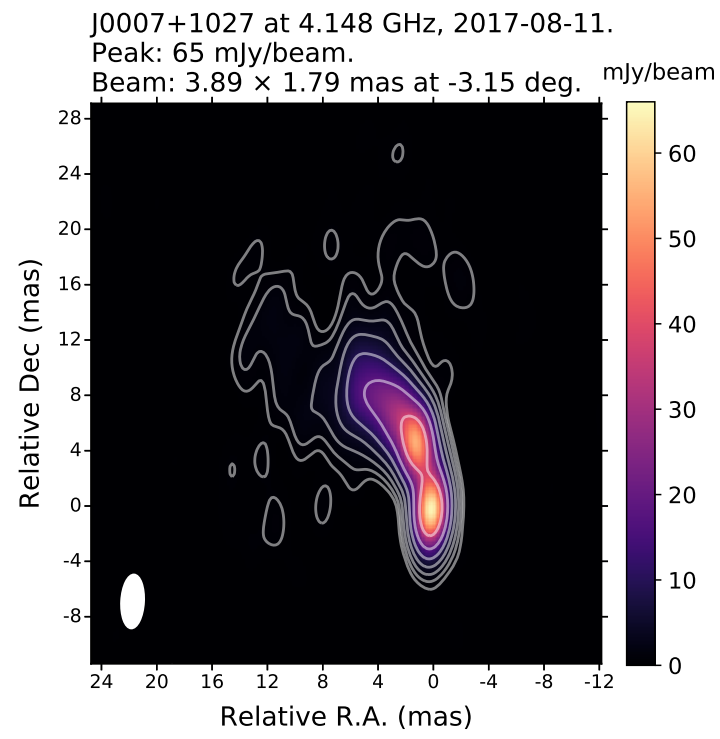

(a)

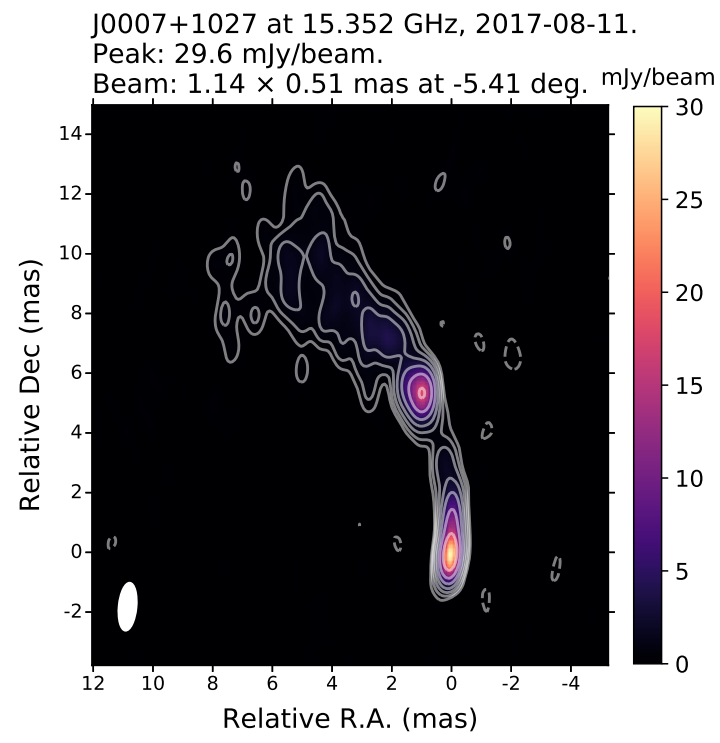

(c)

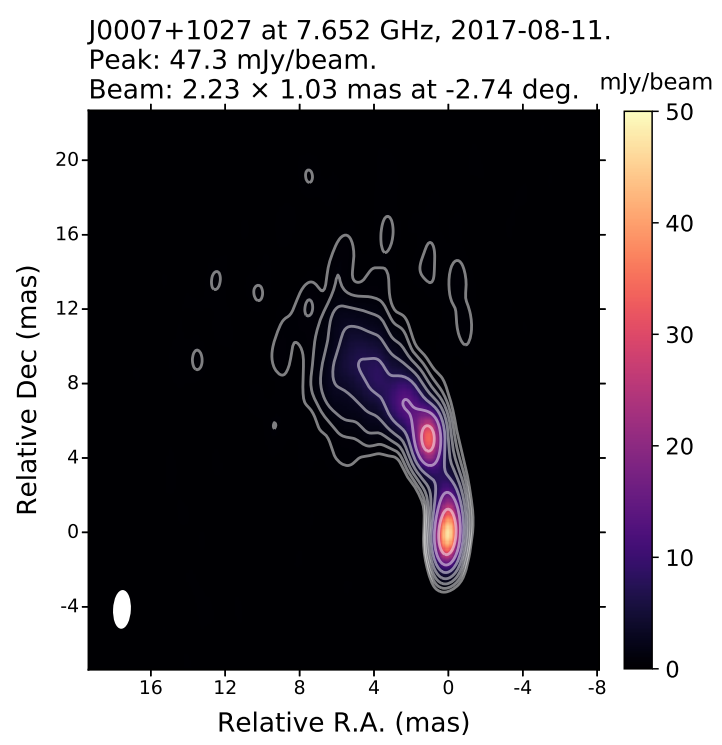

(b)

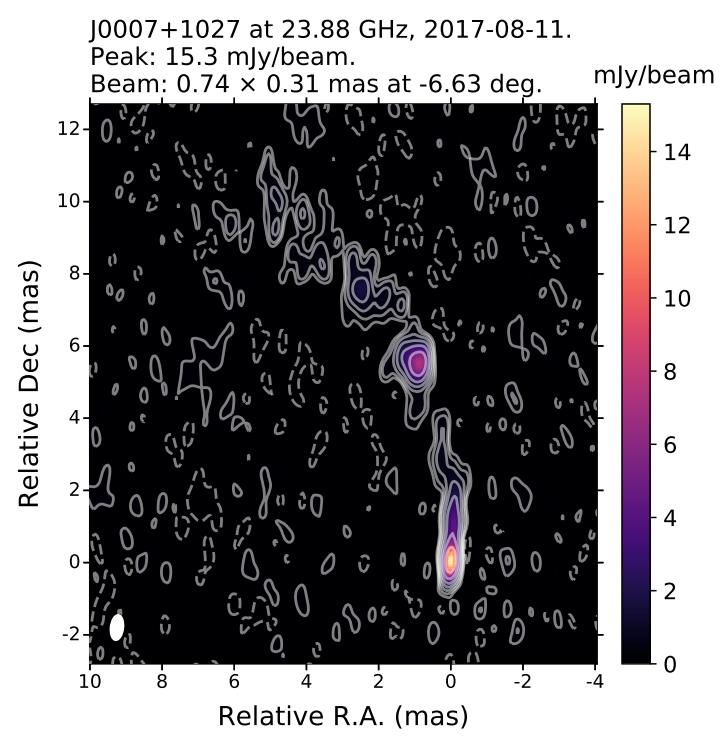

(d)

Fig. B.2. Self-calibrated images of J0007+1027. The images were obtained at (a) $4.148 \mathrm{GHz}$, (b) $7.652 \mathrm{GHz}$, (c) $15.352 \mathrm{GHz}$ and (d) $23.88 \mathrm{GHz}$. The rms noise level from the lower to the higher frequency is $0.2,0.1,0.1,0.09 \mathrm{mJy} \mathrm{beam}^{-1}$. The interferometric beam (ellipse) is displayed on the bottom-left corner of each image. Contours represent $-0.9 \%, 0.9 \%, 1.8 \%, 3.6 \%, 7.2 \%, 14.4 \%, 28.8 \%$, and $57.6 \%$ of the peak intensity at each image. 
W. Chamani et al.: Testing the magnetic flux paradigm for radio loudness of AGN

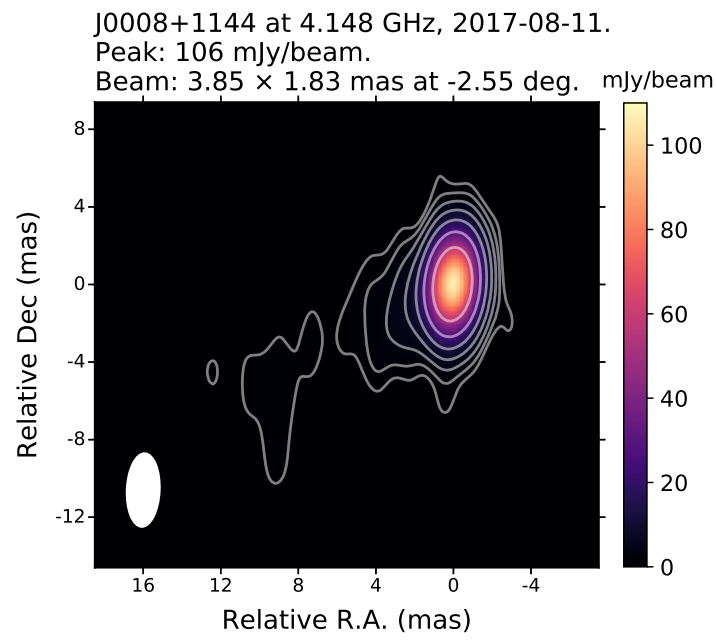

(a)

J0008+1144 at $15.352 \mathrm{GHz}, 2017-08-11$.

Peak: $43.2 \mathrm{mJy} / \mathrm{beam}$.

Beam: $1.09 \times 0.50$ mas at $-4.57 \mathrm{deg} . \mathrm{mJy} / \mathrm{beam}$

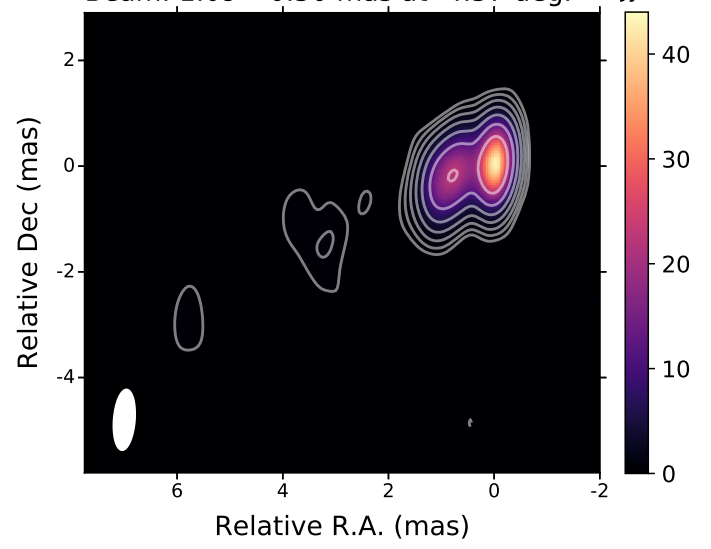

(c)
J0008+1144 at $7.652 \mathrm{GHz}, 2017-08-11$.

Peak: $69 \mathrm{mJy} / \mathrm{beam}$.

Beam: $2.15 \times 1.01$ mas at -1.76 deg. $\mathrm{mJy} / \mathrm{beam}$

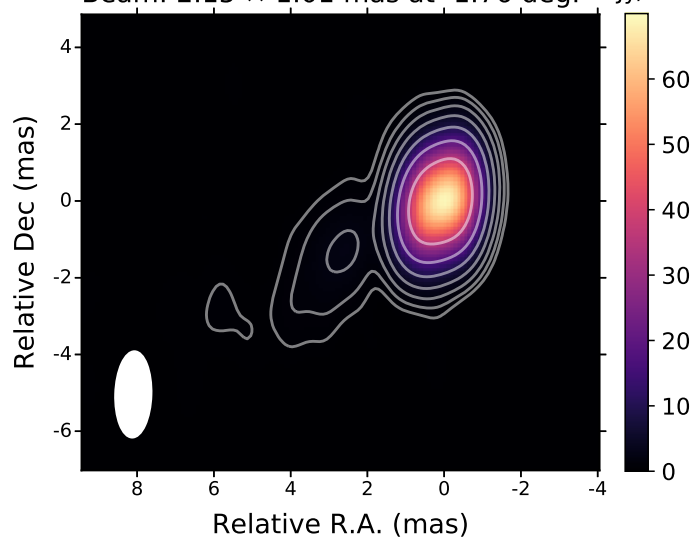

(b)

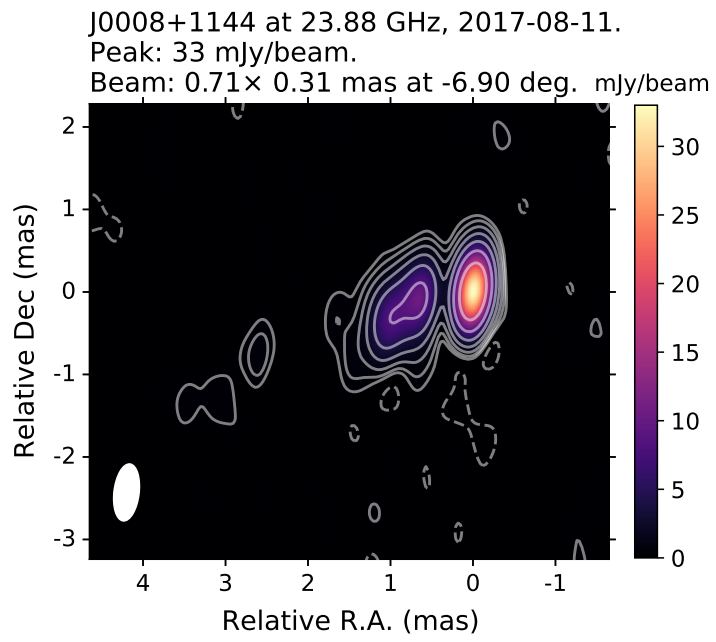

(d)

Fig. B.3. Self-calibrated images of J0008+1144. The images were obtained at (a) $4.148 \mathrm{GHz}$, (b) $7.652 \mathrm{GHz}$, (c) $15.352 \mathrm{GHz}$ and (d) $23.88 \mathrm{GHz}$. The rms noise level from the lower to the higher frequency is $0.2,0.1,0.1,0.1$ mJy beam $^{-1}$. The interferometric beam (ellipse) is displayed on the bottom-left corner of each image. Contours represent $-0.8 \%, 0.8 \%, 1.6 \%, 3.2 \%, 6.4 \%, 12.8 \%, 25.6 \%$, and $51.2 \%$ of the peak intensity at each image. 


\section{Appendix C: Core spectrum of the calibrators}

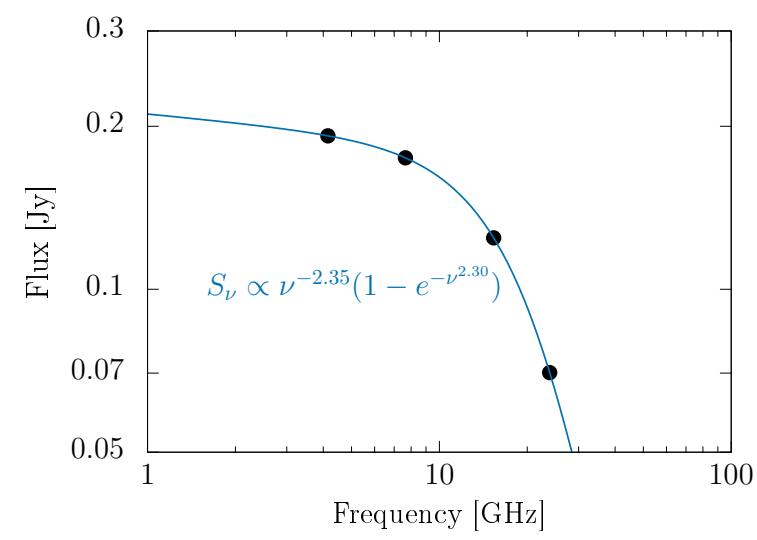

Fig. C.1. Core spectrum of J0006.

The correlated flux density of the calibrators as a function of frequency is shown in Figs. C.1-C.3. The black filled circles represent the core for all cases; the blue curves are the fits. The spectrum of J0006 is fitted with a function of the form $S_{v} \propto v^{i}\left(1-e^{-\nu^{j-i}}\right)$. On the other hand, the spectra of J0007 and J0008 are fitted with a function of the form $S_{v} \propto v^{i}$. Both the core and the bright component 5 mas to the north of the core in J0007 are fully optically thin regions. The spectrum of J0008 appears to be composed of both a rising and a declining part. Further observations are needed to determine the spectral index of the rising side for both J0006 and J0008.

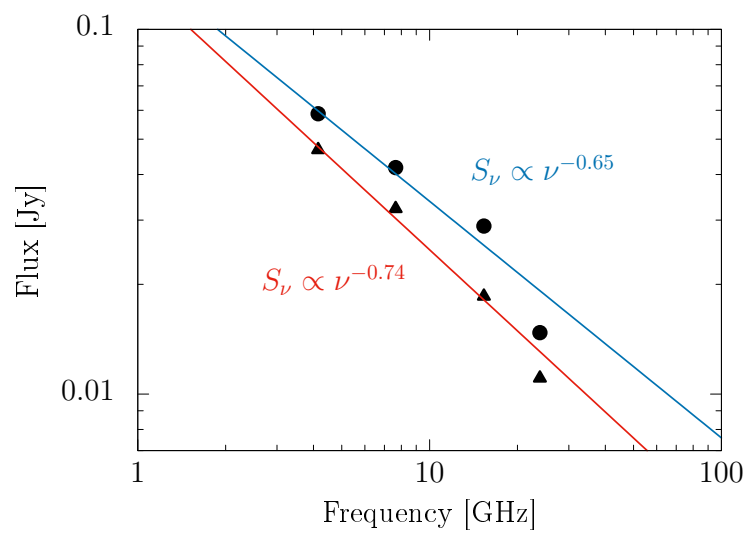

Fig. C.2. Core spectrum of J0007. The black triangle represents the bright knot 5 mas to the north of the core.

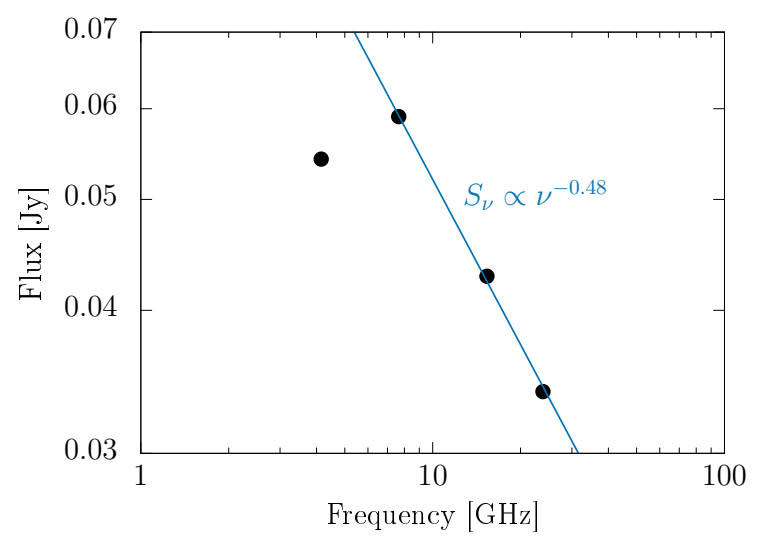

Fig. C.3. Core spectrum of J0008. 


\section{Appendix D: Self- and phase-referencing errors}

\section{D.1. Self-referencing core-shift errors}

Table D.1. Image shift errors.

\begin{tabular}{|c|c|c|c|c|c|c|}
\hline \multirow[t]{2}{*}{ Frequency } & \multicolumn{2}{|c|}{ J0006 } & \multicolumn{2}{|c|}{ J0007 } & \multicolumn{2}{|c|}{$\mathrm{J} 0008$} \\
\hline & $\begin{array}{c}\text { RA } \\
\text { (mas) }\end{array}$ & $\begin{array}{c}\text { Dec } \\
\text { (mas) }\end{array}$ & $\begin{array}{c}\mathrm{RA} \\
\text { (mas) }\end{array}$ & $\begin{array}{c}\text { Dec } \\
\text { (mas) }\end{array}$ & $\begin{array}{c}\text { RA } \\
\text { (mas) }\end{array}$ & $\begin{array}{c}\text { Dec } \\
\text { (mas) }\end{array}$ \\
\hline$C-X$ & 0.029 & 0.026 & 0.026 & 0.026 & 0.031 & 0.028 \\
\hline$X-U$ & 0.014 & 0.013 & 0.013 & 0.014 & 0.015 & 0.014 \\
\hline$U-K$ & 0.008 & 0.008 & 0.008 & 0.008 & 0.009 & 0.009 \\
\hline
\end{tabular}

Table D.2. Core position errors

\begin{tabular}{|c|c|c|c|c|c|c|}
\hline \multirow[t]{2}{*}{ Frequency } & \multicolumn{2}{|c|}{ J0006 } & \multicolumn{2}{|c|}{ J0007 } & \multicolumn{2}{|c|}{ J0008 } \\
\hline & $\begin{array}{c}\mathrm{RA} \\
(\mathrm{mas})\end{array}$ & $\begin{array}{c}\text { Dec } \\
\text { (mas) }\end{array}$ & $\begin{array}{c}\mathrm{RA} \\
(\mathrm{mas})\end{array}$ & $\begin{array}{c}\text { Dec } \\
(\mathrm{mas})\end{array}$ & $\begin{array}{c}\mathrm{RA} \\
(\mathrm{mas})\end{array}$ & $\begin{array}{c}\text { Dec } \\
\text { (mas) }\end{array}$ \\
\hline $\bar{C}$ & 0.003 & 0.006 & 0.017 & 0.028 & 0.075 & 0.045 \\
\hline$X$ & 0.010 & 0.006 & 0.015 & 0.015 & 0.009 & 0.009 \\
\hline$U$ & 0.006 & 0.003 & 0.006 & 0.012 & 0.003 & 0.006 \\
\hline$K$ & 0.005 & 0.005 & 0.003 & 0.004 & 0.002 & 0.005 \\
\hline
\end{tabular}

Table D.3. Core identification errors.

\begin{tabular}{|c|c|c|c|c|c|c|}
\hline \multirow[t]{2}{*}{ Frequency } & \multicolumn{2}{|c|}{ J0006 } & \multicolumn{2}{|c|}{ J0007 } & \multicolumn{2}{|c|}{ J0008 } \\
\hline & $\begin{array}{c}\mathrm{RA} \\
\text { (mas) }\end{array}$ & $\begin{array}{c}\text { Dec } \\
\text { (mas) }\end{array}$ & $\begin{array}{c}\mathrm{RA} \\
\text { (mas) }\end{array}$ & $\begin{array}{c}\text { Dec } \\
(\mathrm{mas})\end{array}$ & $\begin{array}{c}\mathrm{RA} \\
\text { (mas) }\end{array}$ & $\begin{array}{c}\text { Dec } \\
\text { (mas) }\end{array}$ \\
\hline$C$ & 0.044 & 0.037 & 0.016 & 0.042 & 0.050 & 0.011 \\
\hline$X$ & 0.012 & 0.014 & 0.009 & 0.022 & 0.021 & 0.019 \\
\hline$U$ & 0.015 & 0.017 & 0.000 & 0.004 & 0.010 & 0.007 \\
\hline$K$ & 0.002 & 0.014 & 0.006 & 0.005 & 0.002 & 0.004 \\
\hline
\end{tabular}

\section{D.2. Phase-referencing core-shift errors}

Table D.4. Thermal errors.

\begin{tabular}{|c|c|c|c|c|c|c|}
\hline \multirow[t]{2}{*}{ Frequency } & \multicolumn{2}{|c|}{ J0006 } & \multicolumn{2}{|c|}{ J0007 } & \multicolumn{2}{|c|}{ J0008 } \\
\hline & $\begin{array}{c}\mathrm{RA} \\
(\mathrm{mas})\end{array}$ & $\begin{array}{c}\text { Dec } \\
(\mathrm{mas})\end{array}$ & $\begin{array}{c}\mathrm{RA} \\
\text { (mas) }\end{array}$ & $\begin{array}{c}\text { Dec } \\
\text { (mas) }\end{array}$ & $\begin{array}{c}\mathrm{RA} \\
\text { (mas) }\end{array}$ & $\begin{array}{c}\text { Dec } \\
\text { (mas) }\end{array}$ \\
\hline$C$ & 0.012 & 0.025 & 0.016 & 0.036 & 0.011 & 0.023 \\
\hline$X$ & 0.005 & 0.011 & 0.018 & 0.039 & 0.013 & 0.028 \\
\hline$U$ & 0.003 & 0.007 & 0.005 & 0.011 & 0.004 & 0.009 \\
\hline$K$ & 0.003 & 0.006 & 0.003 & 0.007 & 0.002 & 0.005 \\
\hline
\end{tabular}


Table D.5. Core identification errors.

\begin{tabular}{|c|c|c|c|c|c|c|}
\hline \multirow[t]{2}{*}{ Frequency } & \multicolumn{2}{|c|}{ J0006 } & \multicolumn{2}{|c|}{ J0007 } & \multicolumn{2}{|c|}{ J0008 } \\
\hline & $\begin{array}{c}\mathrm{RA} \\
(\mathrm{mas})\end{array}$ & $\begin{array}{c}\text { Dec } \\
(\mathrm{mas})\end{array}$ & $\begin{array}{c}\mathrm{RA} \\
\text { (mas) }\end{array}$ & $\begin{array}{c}\text { Dec } \\
\text { (mas) }\end{array}$ & $\begin{array}{c}\mathrm{RA} \\
(\mathrm{mas})\end{array}$ & $\begin{array}{c}\text { Dec } \\
\text { (mas) }\end{array}$ \\
\hline$C$ & 0.014 & 0.013 & 0.003 & 0.101 & 0.070 & 0.019 \\
\hline$X$ & 0.007 & 0.009 & 0.017 & 0.004 & 0.052 & 0.060 \\
\hline$U$ & 0.008 & 0.019 & 0.002 & 0.016 & 0.019 & 0.003 \\
\hline$K$ & 0.008 & 0.020 & 0.017 & 0.003 & 0.006 & 0.016 \\
\hline
\end{tabular}

Table D.6. Estimated errors for the time-variable part of the tropospheric delay gradient between the target and phase reference calibrator.

\begin{tabular}{|c|c|c|c|c|c|c|}
\hline \multirow[t]{2}{*}{ Frequency } & \multicolumn{2}{|c|}{ J0006 } & \multicolumn{2}{|c|}{$\mathrm{J} 0007$} & \multicolumn{2}{|c|}{$\mathrm{J} 0008$} \\
\hline & $\begin{array}{c}\mathrm{RA} \\
(\mathrm{mas})\end{array}$ & $\begin{array}{c}\text { Dec } \\
\text { (mas) }\end{array}$ & $\begin{array}{c}\mathrm{RA} \\
\text { (mas) }\end{array}$ & $\begin{array}{c}\text { Dec } \\
(\mathrm{mas})\end{array}$ & $\begin{array}{c}\mathrm{RA} \\
\text { (mas) }\end{array}$ & $\begin{array}{c}\text { Dec } \\
\text { (mas) }\end{array}$ \\
\hline$C-X$ & 0 & 0 & 0 & 0 & 0 & 0 \\
\hline$X-U$ & 0.004 & 0.007 & 0.002 & 0.003 & 0.003 & 0.004 \\
\hline$U-K$ & 0.010 & 0.016 & 0.004 & 0.007 & 0.005 & 0.008 \\
\hline
\end{tabular}

Notes. The values are given per source and per frequency pair. Since $C$ and $X$ band observations were simultaneous, the non-dispersive tropospheric delay error vanishes when a difference between the frequencies is taken; hence, $C-X$ error is zero.

Table D.7. Estimated errors due to residual ionospheric delay gradient between the target source and phase reference calibrator.

\begin{tabular}{|c|c|c|c|c|c|c|}
\hline \multirow[t]{2}{*}{ Frequency } & \multicolumn{2}{|c|}{ J0006 } & \multicolumn{2}{|c|}{ J0007 } & \multicolumn{2}{|c|}{ J0008 } \\
\hline & $\begin{array}{c}\mathrm{RA} \\
(\mathrm{mas})\end{array}$ & $\begin{array}{c}\text { Dec } \\
\text { (mas) }\end{array}$ & $\begin{array}{c}\mathrm{RA} \\
\text { (mas) }\end{array}$ & $\begin{array}{c}\text { Dec } \\
\text { (mas) }\end{array}$ & $\begin{array}{c}\mathrm{RA} \\
\text { (mas) }\end{array}$ & $\begin{array}{c}\text { Dec } \\
\text { (mas) }\end{array}$ \\
\hline $\bar{C}$ & 0.038 & 0.082 & 0.016 & 0.035 & 0.020 & 0.042 \\
\hline$X$ & 0.012 & 0.025 & 0.005 & 0.011 & 0.006 & 0.013 \\
\hline$U$ & 0.003 & 0.006 & 0.001 & 0.003 & 0.001 & 0.003 \\
\hline$K$ & 0.001 & 0.003 & 0.001 & 0.001 & 0.001 & 0.001 \\
\hline
\end{tabular}

Notes. Values are given per source and per frequency. 


\section{Appendix E: Spectral index maps after alignment}

J0006+1235: smooth spectral index gradients along the jet are seen in all frequency pairs (Fig. F.1). The core is located near the optically thick region for the $4.15-7.65 \mathrm{GHz}$ section with highest spectral index in the most upstream region. At higher frequencies the core becomes first flat and then slightly optically thin. This result agrees with the core spectrum index shown in Fig. C.1

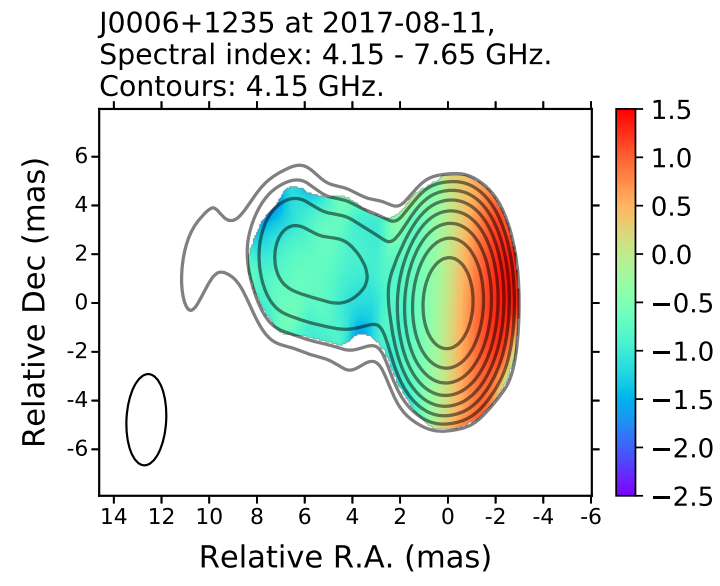

(a)
J0007+1027: interestingly the core is optically thin in all images (Fig. F.2). This result agrees with the steep spectrum seen in both the core and the northern knot in Fig. C.2.

J0008+1144: smooth spectral index gradients along the jet are detected in all frequency pairs (Fig. F.3). The core is found to be the optically thick region only for the $4.15-7.65 \mathrm{GHz}$ section. At higher frequencies, the maps show flat or optically thin spectral index. These results also agree with the core spectrum shown in Fig. C.3, which comprises of a rising ( $C-X$ bands $)$ and a declining part ( $X-K$ bands).

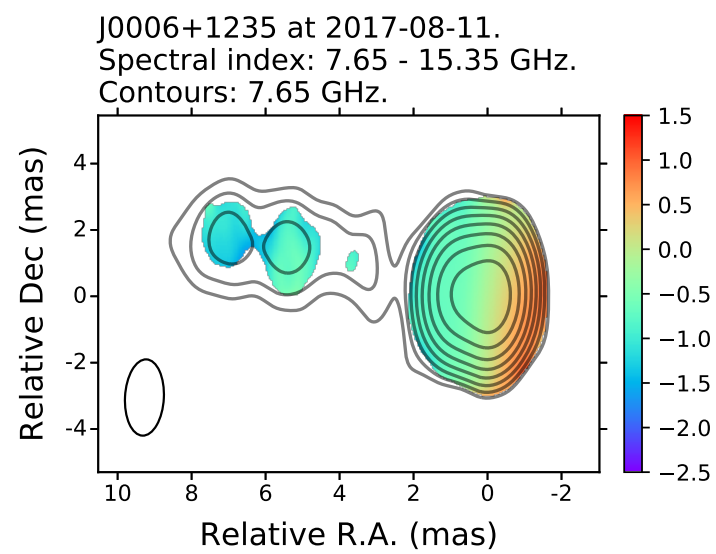

(b)

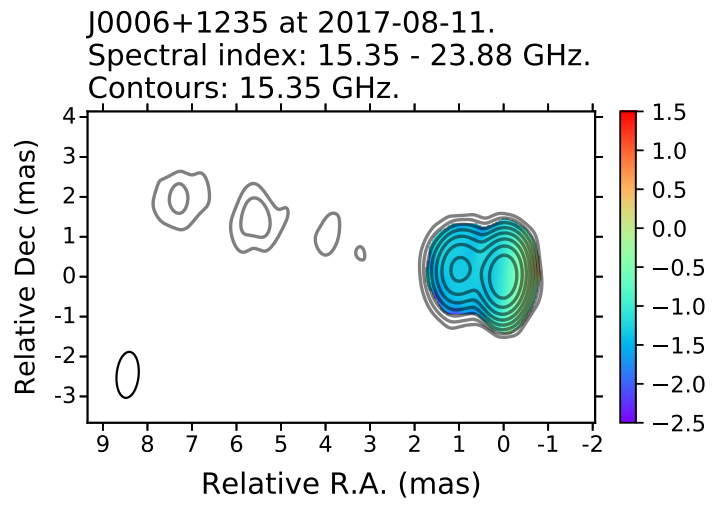

(c)

Fig. E.1. Spectral index maps for J0006 per frequency pair. The spectral index is indicated in the colourbar. The aligned images correspond to 4.15-7.65 GHz, 7.65-15.35 GHz and $15.35-23.88 \mathrm{GHz}$, respectively. The interferometric beam (ellipse) is displayed on the bottom-left corner of each image. 


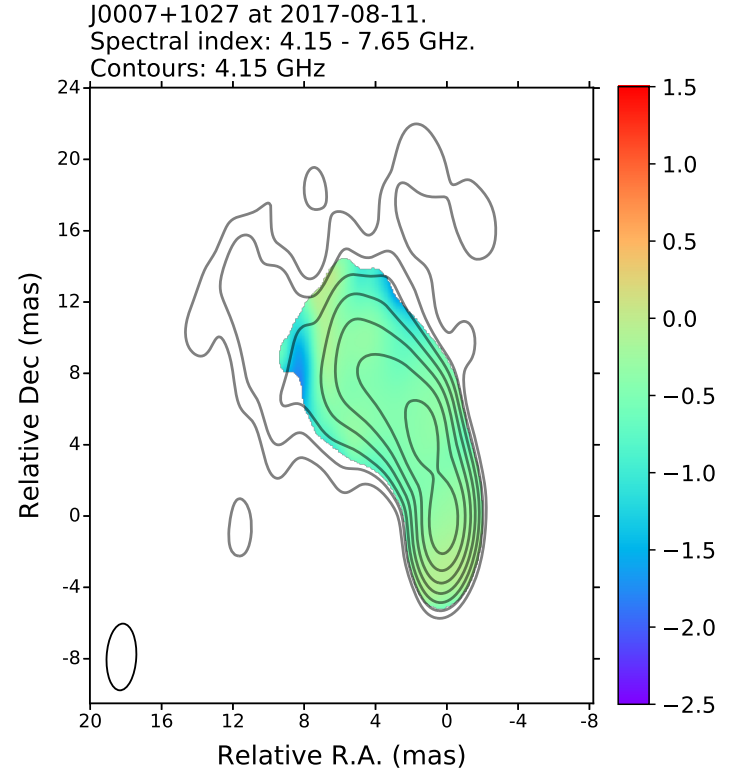

(a)

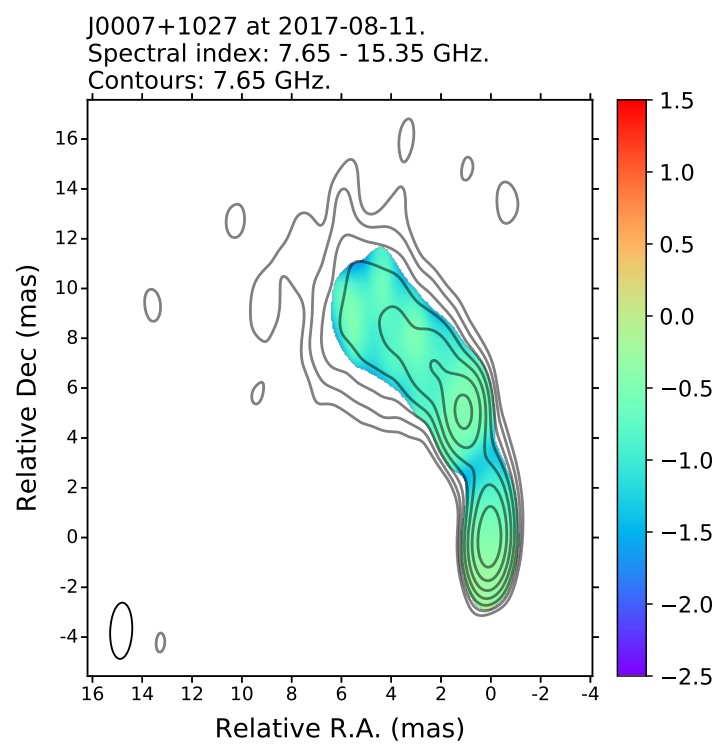

(b)

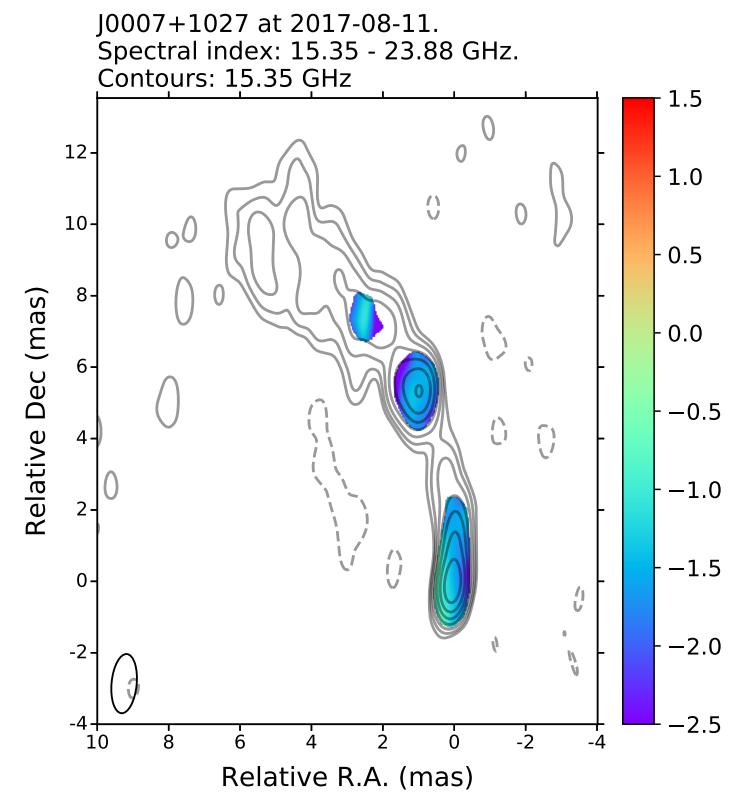

(c)

Fig. E.2. Spectral index maps for J0007 per frequency pair. The spectral index is indicated in the colourbar. The aligned images correspond to $4.15-7.65 \mathrm{GHz}, 7.65-15.35 \mathrm{GHz}$ and $15.35-23.88 \mathrm{GHz}$, respectively. The interferometric beam (ellipse) is displayed on the bottom-left corner of each image. 


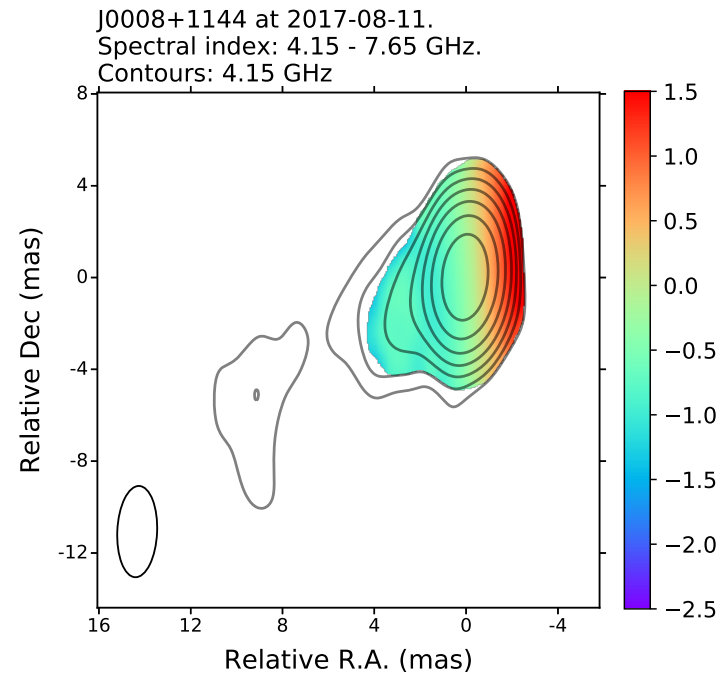

(a)

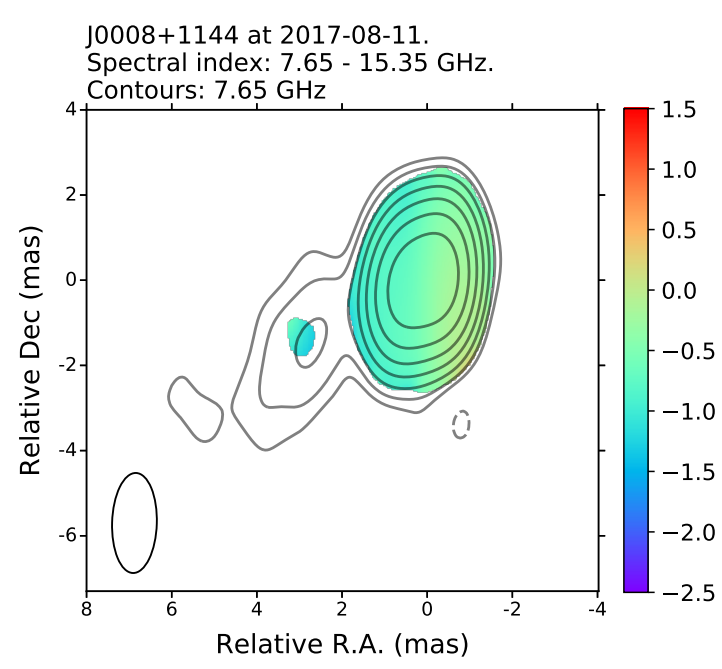

(b)

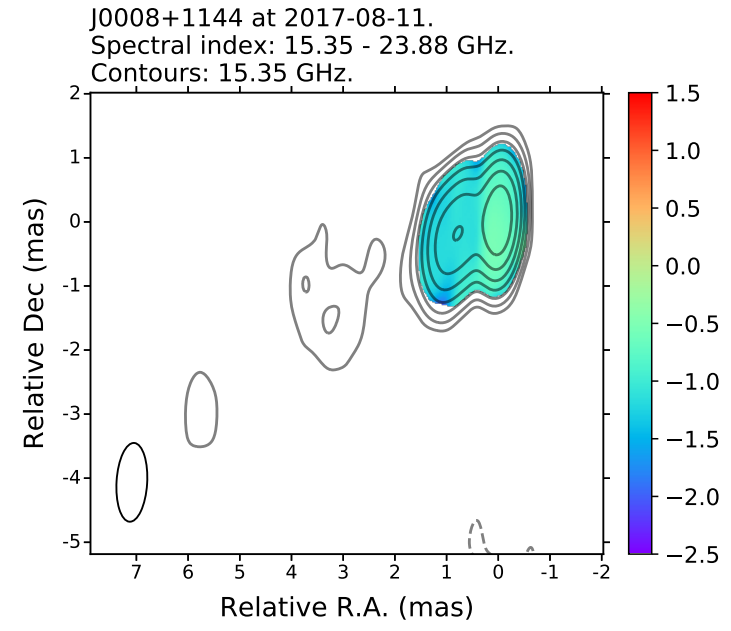

(c)

Fig. E.3. Spectral index maps for J0008 per frequency pair. The spectral index is indicated in the colourbar. The aligned images correspond to 4.15-7.65 GHz, 7.65-15.35 GHz and $15.35-23.88 \mathrm{GHz}$, respectively. The interferometric beam (ellipse) is displayed on the bottom-left corner of each image. 


\section{Appendix F: Projected core-shifts of III Zw 2 with each calibrator}

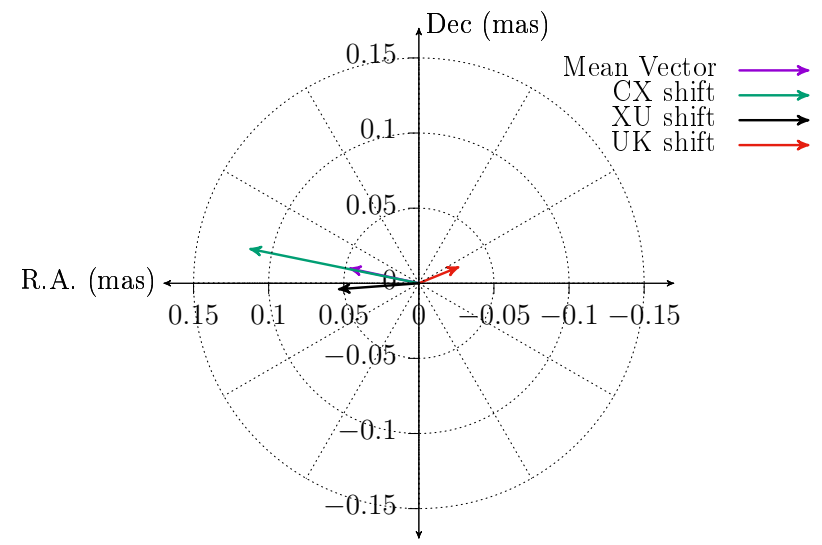

(a)

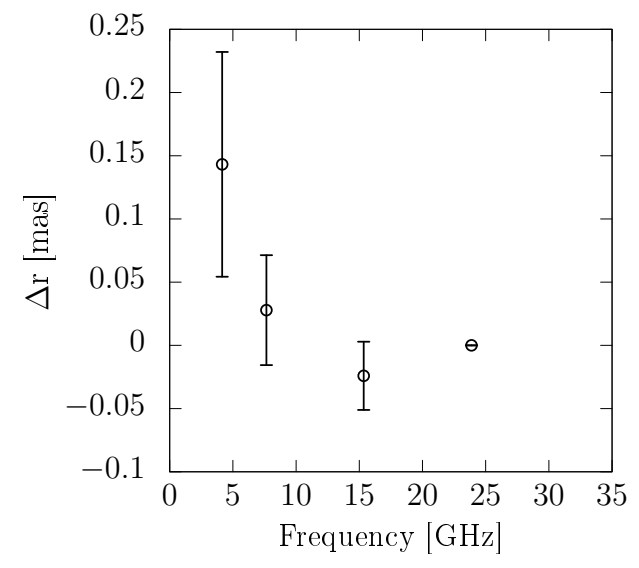

(b)

Fig. F.1. (a) Core-shift vectors of III Zw 2 with calibrator J0006, (b) Core-shift values with $K$ band as the reference frequency.

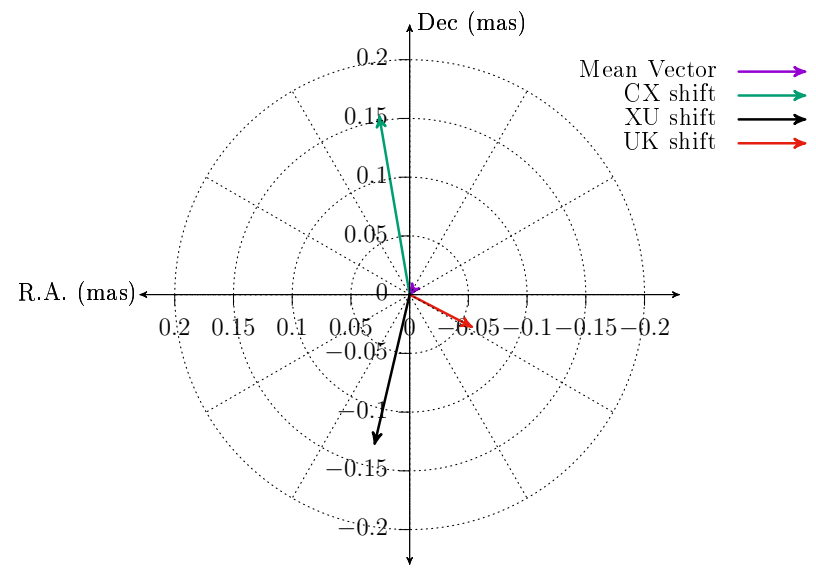

(a)

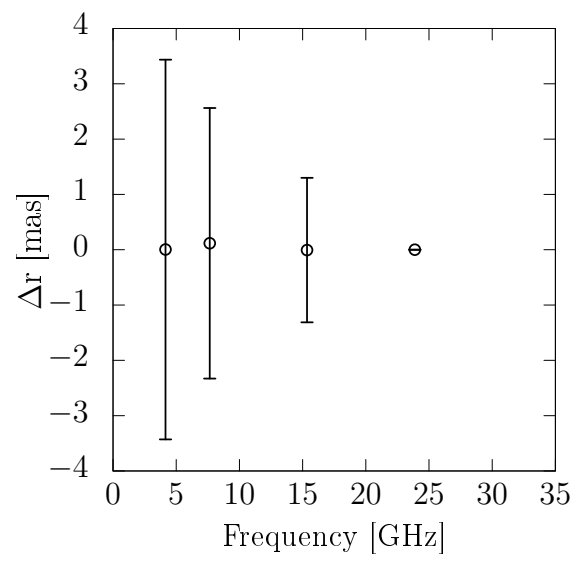

(b)

Fig. F.2. (a) Core-shift vectors of III Zw 2 with calibrator J0007, (b) Core-shift values with $K$ band as the reference frequency.

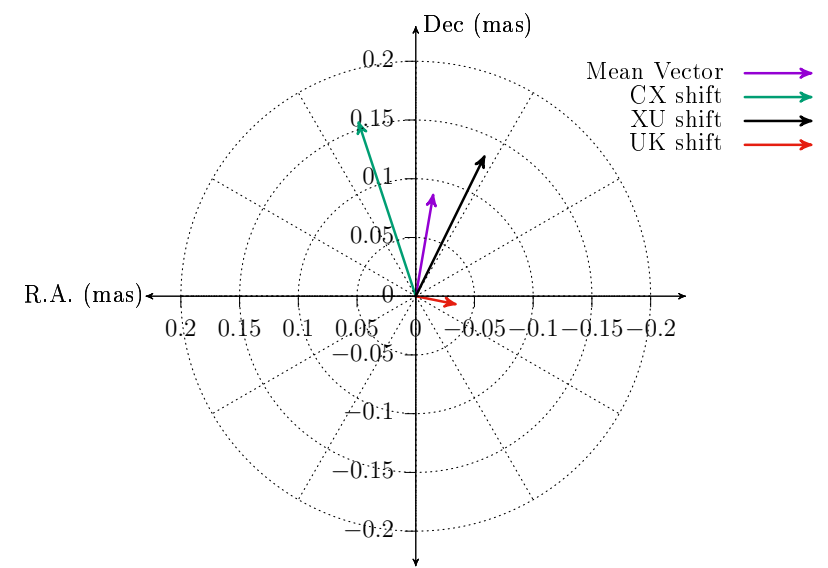

(a)

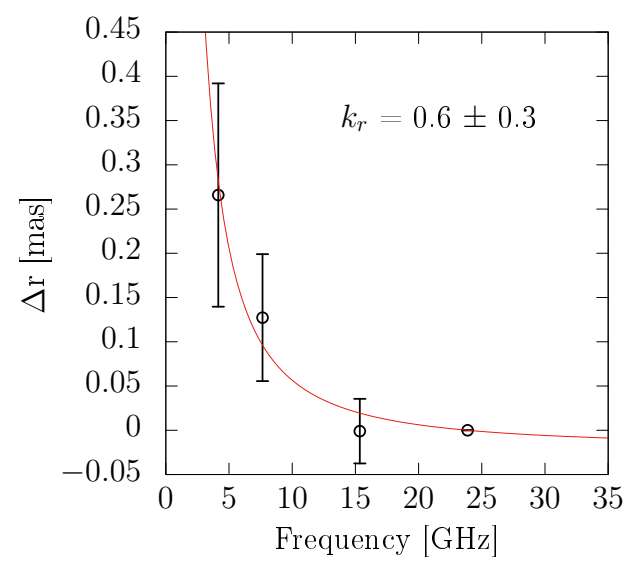

(b)

Fig. F.3. (a) Core-shift vectors of III Zw 2 with calibrator J0008, (b) Core-shift values with $K$ band as the reference frequency. The red curve indicates the best fit of the form: $\Delta r=a\left(v_{\mathrm{GHz}}^{-1 / k_{r}}-23.88^{-1 / k_{r}}\right)$. The fitting parameters are $a=2.8 \pm 2.9$ and $k_{r}=0.6 \pm 0.3$. 